\title{
Viscosity Approximation Method for System of Variational Inclusions Problems and Fixed-Point Problems of a Countable Family of Nonexpansive Mappings
}

\author{
Chaichana Jaiboon ${ }^{1}$ and Poom Kumam ${ }^{2}$ \\ ${ }^{1}$ Department of Mathematics, Faculty of Liberal Arts, Rajamangala University of \\ Technology Rattanakosin (RMUTR), Bangkok 10100, Thailand \\ ${ }^{2}$ Department of Mathematics, Faculty of Science, King Mongkut's University of \\ Technology Thonburi (KMUTT), Bangkok 10140, Thailand
}

Correspondence should be addressed to Chaichana Jaiboon, chaichana.j@rmutr.ac.th and Poom Kumam, poom.kum@kmutt.ac.th

Received 22 January 2012; Accepted 2 March 2012

Academic Editor: Yonghong Yao

Copyright (C) 2012 C. Jaiboon and P. Kumam. This is an open access article distributed under the Creative Commons Attribution License, which permits unrestricted use, distribution, and reproduction in any medium, provided the original work is properly cited.

We propose new iterative schemes for finding the common element of the set of common fixed points of countable family of nonexpansive mappings, the set of solutions of the variational inequality problem for relaxed cocoercive and Lipschitz continuous, the set of solutions of system of variational inclusions problem, and the set of solutions of equilibrium problems in a real Hilbert space by using the viscosity approximation method. We prove strong convergence theorem under some parameters. The results in this paper unify and generalize some well-known results in the literature.

\section{Introduction}

Let $C$ be a nonempty closed convex subset of a real Hilbert space $H$. A mapping $S$ of $C$ into itself is called nonexpansive if $\|S x-S y\| \leq\|x-y\|$ for all $x, y \in C$. We denote by $F(S)$ the set of fixed points of $S$; that is, $F(S)=\{x \in C: S x=x\}$. If $C \subset H$ is nonempty, closed and convex and let $S: C \rightarrow C$ be a nonexpansive mapping, then $F(S)$ is closed and convex and $F(S) \neq \emptyset$, when $C$ is bounded; see, for example, $[1,2]$. The metric projection, $P_{C}$, onto a given nonempty, closed and convex subset $C$, satisfies the nonexpansive with $F\left(P_{C}\right)=C$. A mapping $B: C \rightarrow C$ is called monotone if $\langle B x-B y, x-y\rangle \geq 0$ for all $x, y \in C$. A mapping $B: C \rightarrow C$ is called $\beta$-inverse-strongly monotone if there exists a constant $\beta>0$ such that 
$\langle B x-B y, x-y\rangle \geq \beta\|x-y\|^{2}$, for all $x, y \in C$. A mapping $B: C \rightarrow C$ is called relaxed $(\phi, \omega)$-cocoercive if there exists $\phi, \omega>0$ such that

$$
\langle B x-B y, x-y\rangle \geq(-\phi)\|B x-B y\|^{2}+\omega\|x-y\|^{2}, \quad \forall x, y \in C .
$$

A mapping $B: C \rightarrow C$ is said to be $\xi$-Lipschitz continuous if there exists $\xi \geq 0$ such that

$$
\|B x-B y\| \leq \xi\|x-y\|, \quad \forall x, y \in C
$$

Let $B: H \rightarrow H$ be a single-valued nonlinear mapping and $M: H \rightarrow 2^{H}$ a multivalued mapping. The variational inclusion problem is to find $\tilde{x} \in H$ such that

$$
\theta \in B(\tilde{x})+M(\tilde{x})
$$

where $\theta$ is the zero vector in $H$. The set of solutions of problem (1.3) is denoted by $I(B, M)$. If $M=\partial \psi_{C}$, where $C$ is a nonempty closed convex subset of $H$ and $\partial \psi_{C}: H \rightarrow[0,+\infty]$ is the indicator function of $C$; that is,

$$
\psi_{C}(x)= \begin{cases}0, & x \in C, \\ +\infty, & x \notin C,\end{cases}
$$

then, the variational inclusion problem (1.3) is equivalent to the variational inequality problems denoted by $\operatorname{VI}(C, B)$ which is to find $\tilde{x} \in C$ such that

$$
\langle B \tilde{x}, y-\tilde{x}\rangle \geq 0, \quad \forall y \in C
$$

In 2003, Takahashi and Toyoda [3] to find $x^{*} \in F(S) \cap \operatorname{VI}(C, B)$ introduced the following iterative scheme:

$$
\begin{gathered}
x_{0} \in \mathrm{C} \text { chosen arbitrary, } \\
x_{n+1}=\alpha_{n} x_{n}+\left(1-\alpha_{n}\right) S P_{C}\left(x_{n}-\xi_{n} B x_{n}\right), \quad \forall n \geq 0,
\end{gathered}
$$

where $B$ is a $\beta$-inverse-strongly monotone mapping, $\left\{\alpha_{n}\right\}$ is a sequence in $(0,1)$, and $\left\{\xi_{n}\right\}$ is a sequence in $(0,2 \beta)$. They showed that if $F(S) \cap \operatorname{VI}(C, B)$ is nonempty, then the sequence $\left\{x_{n}\right\}$ generated by (1.6) converges weakly to some $x^{*} \in F(S) \cap \operatorname{VI}(C, B)$.

In 2008, Zhang et al. [4] to find $x^{*} \in F(S) \cap I(M, B)$. They introduced the following new iterative scheme:

$$
\begin{gathered}
x_{0} \in C \text { chosen arbitrary, } \\
y_{n}=J_{M, \lambda}\left(x_{n}-\lambda B x_{n}\right), \\
x_{n+1}=\alpha_{n} x+\left(1-\alpha_{n}\right) S y_{n}, \quad \forall n \geq 0,
\end{gathered}
$$

where $J_{M, \lambda}=(I+\lambda M)^{-1}$ is the resolvent operator associated with $M$ and a positive number $\lambda,\left\{\alpha_{n}\right\}$ is a sequence in the interval $[0,1]$. 
Let $F$ be a bifunction of $C \times C$ into $\mathbb{R}$, where $\mathbb{R}$ is the set of real numbers. The equilibrium problem for $F: C \times C \rightarrow \mathbb{R}$ is to find $\tilde{x} \in C$ such that

$$
F(\tilde{x}, y) \geq 0, \quad \forall y \in C
$$

The set of solutions of (1.8) is denoted by $\mathrm{EP}(F)$. Many problems in applied sciences, such as monotone inclusion problems, variational inequality problems, saddle point problems, Nash equilibria in noncooperative games, as well as certain fixed-point problems reduce to finding some element to $\mathrm{EP}(F)$ in Hilbert and Banach spaces (see [5-14]).

Given any $r>0$. The operator $T_{r}: H \rightarrow C$ defined by

$$
T_{r}(x)=\left\{z \in C: F(z, y)+\frac{1}{r}\langle y-z, z-x\rangle \geq 0, \forall y \in C\right\},
$$

is called the resolvent of $F$ (see $[5,6]$ ).

It is shown in [6] that, under suitable hypotheses on $F$ (to be stated precisely in Section 2), $T_{r}: H \rightarrow C$ is single valued and firmly nonexpansive and satisfies

$$
F\left(T_{r}\right)=\mathrm{EP}(F), \quad \forall r>0 .
$$

Using this result, for finding an element of $F(S) \cap \operatorname{VI}(C, B) \cap \operatorname{EP}(F)$, Su et al. [15] introduced the following iterative scheme by the viscosity approximation method in Hilbert spaces:

$$
\begin{gathered}
x_{0} \in C \text { chosen arbitrary, } \\
x_{n+1}=\alpha_{n} f\left(x_{n}\right)+\left(1-\alpha_{n}\right) S P_{C}\left(I-\xi_{n} B\right) T_{r_{n}} x_{n}, \quad \forall n \geq 0,
\end{gathered}
$$

where $f: C \rightarrow C$ is a contraction (i.e., $\|f(x)-f(y)\| \leq \psi\|x-y\|$, for all $x, y \in C$ and $0 \leq$ $\psi<1)$ and $\left\{\alpha_{n}\right\} \subset(0,1), \xi_{n} \subset(0,2 \beta)$, and $r_{n} \subset(0, \infty)$ satisfy some appropriate conditions. Furthermore, they prove $\left\{x_{n}\right\}$ converges strongly to the same point $x^{*} \in F(S) \cap \operatorname{VI}(C, B) \cap$

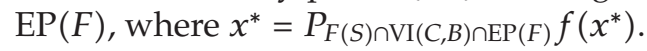

In this paper, motivated and inspired by the above facts, we introduce a new iterative scheme for finding a common element of the set of solutions of the variational inequalities for $\mu$-Lipschitz continuous and relaxed $(\phi, \omega)$-cocoercive mapping, the set of solutions to the variational inclusion for family of $\alpha$-inverse strongly monotone mappings, the set of fixed points of a countable family of nonexpansive mappings, and the set of solutions of an equilibrium problem in a real Hilbert space by using the viscosity approximation method. Strong convergence results are derived under suitable conditions in a real Hilbert space.

\section{Preliminaries}

In this section, we will recall some basic notations and collect some conclusions that will be used in the next section.

Let $H$ be a real Hilbert space whose inner product and norm are denoted by $\langle\cdot, \cdot\rangle$ and $\|\cdot\|$, respectively. We denote strong convergence of $\left\{x_{n}\right\}$ to $x \in H$ by $x_{n} \rightarrow x$ and 
weak convergence by $x_{n} \rightarrow x$. Let $C$ be nonempty closed convex subset of $H$. Recall that for all $x \in H$ there exists a unique nearest point in $C$ to $x$ denoted $P_{C} x$; that is, $\left\|x-P_{C} x\right\| \leq\|x-y\|$, for all $y \in C$. The mapping $P_{C}$ is nonexpansive; that is, $\left\|P_{C} x-P_{C} y\right\| \leq$ $\|x-y\|$, for all $x, y \in H$. The mapping $P_{C}$ is firmly nonexpansive; that is, $\left\|P_{C} x-P_{C} y\right\|^{2} \leq$ $\left\langle P_{C} x-P_{C} y, x-y\right\rangle$, for all $x, y \in H$. It is well known that

$$
\tilde{x} \in \operatorname{VI}(C, B) \Leftrightarrow \tilde{x}=P_{C}(\tilde{x}-\lambda B \tilde{x}), \quad \forall \lambda>0 .
$$

A set-valued mapping $M: H \rightarrow 2^{H}$ is called monotone if, for all $x, y \in H, f \in M x$ and $g \in M y$ imply $\langle x-y, f-g\rangle \geq 0$. A monotone mapping $M: H \rightarrow 2^{H}$ is called maximal, if its graph of any Graph $(M):=\{(x, f) \in H \times H \mid f \in M(x)\}$ of $M$ is not properly contained in the graph of any other monotone mapping. It is well known that a monotone mapping $M$ is maximal if and only if for all $(x, f) \in H \times H,\langle x-y, f-g\rangle \geq 0$, for all $(y, g) \in \operatorname{Graph}(M)$ (the graph of mapping $M$ ) implies that $f \in M x$.

Definition 2.1. Let $M: H \rightarrow 2^{H}$ be a multivalued maximal monotone mapping; then the set-valued mapping $J_{M, \lambda}: H \rightarrow H$ defined by

$$
J_{M, \lambda}(\tilde{x})=(I+\lambda M)^{-1}(\tilde{x}), \quad \forall \tilde{x} \in H,
$$

is called the resolvent operator associated with $M$, where $\lambda$ is any positive number and $I$ is the identity mapping.

Lemma 2.2 (see [16]). Let $M: H \rightarrow 2^{H}$ be a maximal monotone mapping and let $B: H \rightarrow H$ be a Lipschitz continuous mapping. Then the mapping $M+B: H \rightarrow 2^{H}$ is a maximal monotone mapping.

Lemma 2.3 (see $[16,17]$ ).

(1) The resolvent operator $J_{M, \lambda}$ is single valued and nonexpansive for all $\lambda>0$; that is,

$$
\left\|J_{M, \lambda}(x)-J_{M, \lambda}(y)\right\| \leq\|x-y\|, \quad \forall x, y \in H, \forall \curlywedge>0 .
$$

(2) The resolvent operator $J_{M, \lambda}$ is 1-inverse-strongly monotone; that is,

$$
\left\|J_{M, \lambda}(x)-J_{M, \lambda}(y)\right\|^{2} \leq\left\langle x-y, J_{M, \lambda}(x)-J_{M, \lambda}(y)\right\rangle, \quad \forall x, y \in H .
$$

Lemma 2.4 (see [17]).

(1) Let $\tilde{x} \in H$ is a solution of problem (1.3) if and only if $\tilde{x}=J_{M, \lambda}(I-\lambda B)$ for all $\lambda>0$; that is,

$$
I(B, M)=F\left(J_{M, \lambda}(I-\lambda B)\right), \quad \forall \lambda>0
$$

(2) If $\lambda \in[0,2 \beta]$, then $I(B, M)$ is a closed convex subset in $H$. 
Lemma 2.5 (see [18]). Each Hilbert space $H$ satisfies Opial's condition; that is, for any sequence $\left\{x_{n}\right\} \subset H$ with $x_{n} \rightarrow x$, the inequality

$$
\liminf _{n \rightarrow \infty}\left\|x_{n}-x\right\|<\liminf _{n \rightarrow \infty}\left\|x_{n}-y\right\|
$$

holds for each $y \in H$ with $y \neq x$.

Lemma 2.6 (see [19]). Let $\left\{x_{n}\right\}$ and $\left\{z_{n}\right\}$ be bounded sequences in a Banach space $E$, and let $\left\{\beta_{n}\right\}$ be a sequence in $[0,1]$ with $0<\liminf _{n \rightarrow \infty} \beta_{n} \leq \lim \sup _{n \rightarrow \infty} \beta_{n}<1$. Suppose $x_{n+1}=\left(1-\beta_{n}\right) z_{n}+\beta_{n} x_{n}$ for all integers $n \geq 1$ and $\lim \sup _{n \rightarrow \infty}\left(\left\|z_{n+1}-z_{n}\right\|-\left\|x_{n+1}-x_{n}\right\|\right) \leq 0$. Then, $\lim _{n \rightarrow \infty}\left\|z_{n}-x_{n}\right\|=0$.

Lemma 2.7 (see [20]). Assume $\left\{a_{n}\right\}$ is a sequence of nonnegative real numbers such that

$$
a_{n+1} \leq\left(1-b_{n}\right) a_{n}+\delta_{n}, \quad n \geq 0,
$$

where $\left\{b_{n}\right\}$ is a sequence in $(0,1)$ and $\left\{\delta_{n}\right\}$ is a sequence in $\mathbb{R}$ such that

(1) $\sum_{n=1}^{\infty} b_{n}=\infty$,

(2) $\lim \sup _{n \rightarrow \infty} \delta_{n} / b_{n} \leq 0$ or $\sum_{n=1}^{\infty}\left|\delta_{n}\right|<\infty$.

Then $\lim _{n \rightarrow \infty} a_{n}=0$.

Lemma 2.8. Let $H$ be a real Hilbert space. Then hold the following identities:

(i) $\|t x+(1-t) y\|^{2}=t\|x\|^{2}+(1-t)\|y\|^{2}-t(1-t)\|x-y\|^{2}, \forall t \in[0,1], \forall x, y \in H$ ，

(ii) $\|x+y\|^{2} \leq\|x\|^{2}+2\langle y, x+y\rangle, \forall x, y \in H$.

Lemma 2.9 (see [21]). Let $C$ be a nonempty closed subset of a Banach space, and let $\left\{S_{n}\right\}$ be a sequence of mappings of $C$ into itself. Suppose that $\sum_{n=1}^{\infty} \sup \left\{\left\|S_{n+1} z-S_{n} z\right\|: z \in C\right\}<\infty$. Then, for each $y \in C,\left\{S_{n} y\right\}$ converges strongly to some point of $C$. Moreover, let $S$ be a mapping of $C$ into itself defined by

$$
S y=\lim _{n \rightarrow \infty} S_{n} y, \quad \forall y \in C
$$

Then $\lim _{n \rightarrow \infty} \sup \left\{\left\|S z-S_{n} z\right\|: z \in C\right\}=0$.

For solving the equilibrium problem for a bifunction $F: C \times C \rightarrow \mathbb{R}$, let us assume that $F$ satisfies the following conditions:

(A1) $F(x, x)=0$ for all $x \in C$;

(A2) $F$ is monotone, that is, $F(x, y)+F(y, x) \leq 0, \forall x, y \in C$;

(A3) for each $x, y, z \in C, \lim _{t \downarrow 0} F(t z+(1-t) x, y) \leq F(x, y)$;

(A4) for each $x \in C, y \mapsto F(x, y)$ is convex and lower semicontinuous.

Lemma 2.10 (see [5]). Let $C$ be a nonempty closed convex subset of $H$, and let $F$ be a bifunction of $C \times C$ into $\mathbb{R}$ satisfying (A1)-(A4). Let $r>0$ and $x \in H$. Then, there exists $z \in C$ such that

$$
F(z, y)+\frac{1}{r}\langle y-z, z-x\rangle \geq 0, \quad \forall y \in C
$$


Lemma 2.11 (see [6]). Assume that $F: C \times C \rightarrow \mathbb{R}$ satisfies (A1)-(A4). For $r>0$ and $x \in H$, define a mapping $T_{r}: H \rightarrow C$ as follows:

$$
T_{r}(x)=\left\{z \in C: F(z, y)+\frac{1}{r}\langle y-z, z-x\rangle \geq 0, \forall y \in C\right\}
$$

for all $x \in H$. Then, the following hold:

(i) $T_{r}$ is single valued;

(ii) $T_{r}$ is firmly nonexpansive; that is, for any $x, y \in H\left\|T_{r} x-T_{r} y\right\|^{2} \leq\left\langle T_{r} x-T_{r} y, x-y\right\rangle$;

(iii) $F\left(T_{r}\right)=\mathrm{EP}(F)$;

(iv) $\mathrm{EP}(F)$ is closed and convex.

Lemma 2.12 (see [22]). Let $H$ be a Hilbert space and $M$ a maximal monotone on $H$. Then, the following holds:

$$
\left\|J_{M, r} x-J_{M, s} x\right\|^{2} \leq \frac{r-s}{r}\left\langle J_{M, r} x-J_{M, s} x, J_{M, r} x-x\right\rangle, \quad \forall s, r>0, x \in H,
$$

where $J_{M, r}=(I+r M)^{-1}$ and $J_{M, s}=(I+s M)^{-1}$.

\section{Main Results}

In this section, we will use the viscosity approximation method to prove a strong convergence theorem for finding a common element of the set of fixed points of a countable family of nonexpansive mappings, the set of solutions of the variational inequality problem for relaxed cocoercive and Lipschitz continuous mappings, the set of solutions of system of variational inclusions, and the set of solutions of equilibrium problem in a real Hilbert space.

Theorem 3.1. Let $C$ be a nonempty closed convex subset of a real Hilbert space $H$, and let $B: C \rightarrow$ $H$ be relaxed $(\phi, \omega)$-cocoercive and $\mu$-Lipschitz continuous with $\omega>\phi \mu^{2}$, for some $\phi, \omega, \mu>0$. Let $\mathcal{G}=\left\{G_{k}: k=1,2,3, \ldots, N\right\}$ be a finite family of $\beta$-inverse strongly monotone mappings from $C$ into $H$, and let $F$ be a bifunction from $C \times C \rightarrow \mathbb{R}$ satisfying (A1)-(A4). Let $f: C \rightarrow C$ be a contraction with coefficient $\psi(0 \leq \psi<1)$, and let $\left\{S_{n}\right\}$ be a sequence of nonexpansive mappings of $C$ into itself such that

$$
\Omega: \bigcap_{n=1}^{\infty} F\left(S_{n}\right) \cap\left(\bigcap_{k=1}^{N} I\left(G_{k}, M_{k}\right)\right) \cap \operatorname{VI}(C, B) \cap \operatorname{EP}(F) \neq \emptyset .
$$

Let the sequences $\left\{x_{n}\right\}$ and $\left\{y_{n}\right\}$ be generated by

$$
\begin{aligned}
x_{1} & =x \in C \text { chosen arbitrarily, } \\
y_{n} & =J_{M_{N}, \lambda_{N, n}}\left(I-\lambda_{N, n} G_{n}\right) \ldots J_{M_{2}, \lambda_{2, n}}\left(I-\lambda_{2, n} G_{2}\right) J_{M_{1}, \lambda_{1, n}}\left(I-\lambda_{1, n} G_{1}\right) T_{r_{n}} x_{n}, \\
x_{n+1} & =\alpha_{n} f\left(x_{n}\right)+\beta_{n} x_{n}+\gamma_{n} S_{n} P_{C}\left(y_{n}-\xi_{n} B y_{n}\right), \quad \forall n \geq 1
\end{aligned}
$$


where $\left\{\alpha_{n}\right\},\left\{\beta_{n}\right\},\left\{\gamma_{n}\right\} \subset(0,1)$ and $\left\{\xi_{n}\right\},\left\{r_{n}\right\} \subset(0, \infty)$ satisfy the following conditions:

(C1) $\alpha_{n}+\beta_{n}+\gamma_{n}=1$,

(C2) $\lim _{n \rightarrow \infty} \alpha_{n}=0, \sum_{n=1}^{\infty} \alpha_{n}=\infty$,

(C3) $0<\liminf _{n \rightarrow \infty} \beta_{n} \leq \lim \sup _{n \rightarrow \infty} \beta_{n}<1$,

(C4) $\left\{\xi_{n}\right\} \subset[a, b]$ for some $a, b$ with $0 \leq a \leq b \leq 2\left(\omega-\phi \mu^{2}\right) / \mu^{2}$ and $\lim _{n \rightarrow \infty}\left|\xi_{n+1}-\xi_{n}\right|=0$,

(C5) $\left\{\lambda_{k, n}\right\}_{k=1}^{N} \subset[c, d] \subset(0,2 \beta)$ and $\lim _{n \rightarrow \infty}\left|\lambda_{k, n+1}-\lambda_{k, n}\right|=0$, for each $k \in\{1,2, \ldots, N\}$,

(C6) $\liminf _{n \rightarrow \infty} r_{n}>0$ and $\lim _{n \rightarrow \infty}\left|r_{n+1}-r_{n}\right|=0$.

Suppose that $\sum_{n=1}^{\infty} \sup \left\{\left\|S_{n+1} z-S_{n} z\right\|: z \in K\right\}<\infty$ for any bounded subset $K$ of $C$. Let $S$ be a mapping of $C$ into itself defined by $S y=\lim _{n \rightarrow \infty} S_{n} y$ for all $y \in C$ and suppose that $F(S)=$ $\bigcap_{n=1}^{\infty} F\left(S_{n}\right)$. Then, the sequences $\left\{x_{n}\right\}$ and $\left\{y_{n}\right\}$ converge strongly to the same point $x^{*} \in \Omega$, where $x^{*}=P_{\Omega} f\left(x^{*}\right)$.

Proof. First, we prove that the mapping $P_{\Omega} f: H \rightarrow C$ has a unique fixed point.

In fact, since $f: C \rightarrow C$ is a contraction with $\psi \in[0,1)$ and $P_{\Omega} f: H \rightarrow \Omega$ is also a contraction, we obtain

$$
\left\|P_{\Omega} f(x)-P_{\Omega} f(y)\right\| \leq\|f(x)-f(y)\| \leq \psi\|x-y\|, \quad \forall x, y \in C .
$$

Therefore, there exists a unique element $x^{*} \in C$ such that $x^{*}=P_{\Omega} f\left(x^{*}\right)$, where

$$
\Omega: \bigcap_{n=1}^{\infty} F\left(S_{n}\right) \cap\left(\bigcap_{k=1}^{N} I\left(G_{k}, M_{k}\right)\right) \cap \operatorname{VI}(C, B) \cap \operatorname{EP}(F) .
$$

Now, we prove that $\left(I-\xi_{n} B\right)$ is nonexpansive.

Indeed, for any $x, y \in C$, since $B: C \rightarrow H$ is a $\mu$-Lipschitz continuous and relaxed $(\phi, \omega)$-cocoercive mappings with $\omega>\phi \mu^{2}$ and $\xi_{n} \leq 2\left(\omega-\phi \mu^{2}\right) / \mu^{2}$, we obtain

$$
\begin{aligned}
\left\|\left(I-\xi_{n} B\right) x-\left(I-\xi_{n} B\right) y\right\|^{2} & =\left\|(x-y)-\xi_{n}(B x-B y)\right\|^{2} \\
& =\|x-y\|^{2}-2 \xi_{n}\langle x-y, B x-B y\rangle+\xi_{n}^{2}\|B x-B y\|^{2} \\
& \leq\|x-y\|^{2}-2 \xi_{n}\left\{-\phi\|B x-B y\|^{2}+\omega\|x-y\|^{2}\right\}+\xi_{n}^{2}\|B x-B y\|^{2} \\
& \leq\|x-y\|^{2}+2 \xi_{n} \phi \mu^{2}\|x-y\|^{2}-2 \xi_{n} \omega\|x-y\|^{2}+\xi_{n}^{2} \mu^{2}\|x-y\|^{2} \\
& =\left(1+2 \xi_{n} \phi \mu^{2}-2 \xi_{n} \omega+\xi_{n}^{2} \mu^{2}\right)\|x-y\|^{2} \\
& =\left(1-\xi_{n} \mu^{2}\left[\frac{2\left(\omega-\phi \mu^{2}\right)}{\mu^{2}}-\xi_{n}\right]\right)\|x-y\|^{2} \\
& \leq\left(1-\xi_{n} \mu^{2}\left[\frac{2\left(\omega-\phi \mu^{2}\right)}{\mu^{2}}-b\right]\right)\|x-y\|^{2} .
\end{aligned}
$$

Setting

$$
\zeta=\frac{\mu^{2}}{2}\left[\frac{2\left(\omega-\phi \mu^{2}\right)}{\mu^{2}}-b\right]>0,
$$


thus,

$$
\left\|\left(I-\xi_{n} B\right) x-\left(I-\xi_{n} B\right) y\right\|^{2} \leq\left(1-2 \xi_{n} \zeta\right)\|x-y\|^{2} \leq\left(1-\xi_{n} \zeta\right)^{2}\|x-y\|^{2},
$$

which implies that

$$
\left\|\left(I-\xi_{n} B\right) x-\left(I-\xi_{n} B\right) y\right\| \leq\left(1-\xi_{n} \zeta\right)\|x-y\| \leq\|x-y\| .
$$

Hence $\left(I-\xi_{n} B\right)$ is nonexpansive.

We divide the proof of Theorem 3.1 into five steps.

Step 1. We show that the sequence $\left\{x_{n}\right\}$ is bounded.

Now, let $\tilde{x} \in \Omega$ and if $\left\{T_{r_{n}}\right\}$ is a sequence of mappings defined as in Lemma 2.11, then $\tilde{x}=P_{C}\left(\tilde{x}-\lambda_{n} B \tilde{x}\right)=T_{r_{n}} \tilde{x}$, and let $u_{n}=T_{r_{n}} x_{n}$. So, we have

$$
\left\|u_{n}-\tilde{x}\right\|=\left\|T_{r_{n}} x_{n}-T_{r_{n}} \tilde{x}\right\| \leq\left\|x_{n}-\tilde{x}\right\|
$$

For $k \in\{1,2, \ldots, N\}$ and for any positive integer number $n$, we define the operator $\Upsilon_{n}^{k}: C \rightarrow$ $H$ as follows:

$$
\begin{aligned}
& \Upsilon_{n}^{0} x=x, \\
& \Upsilon_{n}^{k} x=J_{M_{k}, \lambda_{k, n}}\left(I-\lambda_{k, n} G_{k}\right) \ldots J_{M_{2,} \lambda_{2, n}}\left(I-\lambda_{2, n} G_{2}\right) J_{M_{1, \lambda}, n}\left(I-\lambda_{1, n} G_{1}\right) x,
\end{aligned}
$$

for all $n$, we get $y_{n}=\Upsilon_{n}^{N} u_{n}$. On the other hand, since $G_{k}: C \rightarrow H$ is $\beta$-inverse strongly monotone and $\lambda_{k, n} \subset[c, d] \subset(0,2 \beta)$, then $J_{M_{k}, \lambda_{k, n}}\left(I-\lambda_{k, n} G_{k}\right)$ is nonexpansive. Thus $\Upsilon_{n}^{k}$ is nonexpansive. From Lemma 2.4(1), we have $\tilde{x}=\Upsilon_{n}^{N} \tilde{x}$. It follows that

$$
\left\|y_{n}-\tilde{x}\right\|=\left\|\Upsilon_{n}^{N} u_{n}-\Upsilon_{n}^{N} \tilde{x}\right\| \leq\left\|u_{n}-\tilde{x}\right\| \leq\left\|x_{n}-\tilde{x}\right\|
$$

Setting $v_{n}=P_{C}\left(y_{n}-\xi_{n} B y_{n}\right)$ and $I-\xi_{n} B$ is a nonexpansive mapping, we obtain

$$
\begin{aligned}
\left\|v_{n}-\tilde{x}\right\| & =\left\|P_{C}\left(y_{n}-\xi_{n} B y_{n}\right)-P_{C}\left(\tilde{x}-\xi_{n} B \tilde{x}\right)\right\| \\
& \leq\left\|\left(y_{n}-\xi_{n} B y_{n}\right)-\left(\tilde{x}-\xi_{n} B \tilde{x}\right)\right\| \\
& =\left\|\left(I-\xi_{n} B\right) y_{n}-\left(I-\xi_{n} B\right) \tilde{x}\right\| \\
& \leq\left\|y_{n}-\tilde{x}\right\| \leq\left\|x_{n}-\tilde{x}\right\| .
\end{aligned}
$$


From (3.2) and (3.12), we deduce that

$$
\begin{aligned}
\left\|x_{n+1}-\tilde{x}\right\| & =\left\|\alpha_{n} f\left(x_{n}\right)+\beta_{n} x_{n}+\gamma_{n} S_{n} v_{n}-\tilde{x}\right\| \\
& \leq \alpha_{n}\left\|f\left(x_{n}\right)-\tilde{x}\right\|+\beta_{n}\left\|x_{n}-\tilde{x}\right\|+\gamma_{n}\left\|v_{n}-\tilde{x}\right\| \\
& \leq \alpha_{n}\left\|f\left(x_{n}\right)-f(\tilde{x})\right\|+\alpha_{n}\|f(\tilde{x})-\tilde{x}\|+\beta_{n}\left\|x_{n}-\tilde{x}\right\|+\gamma_{n}\left\|x_{n}-\tilde{x}\right\| \\
& \leq \alpha_{n} \psi\left\|x_{n}-\tilde{x}\right\|+\alpha_{n}\|f(\tilde{x})-\tilde{x}\|+\left(1-\alpha_{n}\right)\left\|x_{n}-\tilde{x}\right\| \\
& \leq\left(1-\alpha_{n}(1-\psi)\right)\left\|x_{n}-\tilde{x}\right\|+\alpha_{n}\|f(\tilde{x})-\tilde{x}\| \\
& =\left(1-\alpha_{n}(1-\psi)\right)\left\|x_{n}-\tilde{x}\right\|+\alpha_{n}(1-\psi) \frac{\|f(\tilde{x})-\tilde{x}\|}{(1-\psi)} \\
& \leq \max \left\{\left\|x_{n}-\tilde{x}\right\|, \frac{\|f(\tilde{x})-\tilde{x}\|}{1-\psi}\right\} .
\end{aligned}
$$

It follows from induction that

$$
\left\|x_{n}-\tilde{x}\right\| \leq \max \left\{\left\|x_{1}-\tilde{x}\right\|, \frac{\|f(\tilde{x})-\tilde{x}\|}{1-\psi}\right\}, \quad \forall n \geq 1 .
$$

Therefore, $\left\{x_{n}\right\}$ is bounded and hence so are $\left\{v_{n}\right\},\left\{y_{n}\right\},\left\{u_{n}\right\},\left\{B y_{n}\right\}$, and $\left\{S_{n} v_{n}\right\}$.

Step 2. We claim that $\lim _{n \rightarrow \infty}\left\|x_{n+1}-x_{n}\right\|=0$.

By the definition of $T_{r}, u_{n}=T_{r_{n}} x_{n}$ and $u_{n+1}=T_{r_{n+1}} x_{n+1}$, we get

$$
\begin{gathered}
F\left(u_{n}, y\right)+\frac{1}{r_{n}}\left\langle y-u_{n}, u_{n}-x_{n}\right\rangle \geq 0, \quad \forall y \in H, \\
F\left(u_{n+1}, y\right)+\frac{1}{r_{n+1}}\left\langle y-u_{n+1}, u_{n+1}-x_{n+1}\right\rangle \geq 0, \quad \forall y \in H .
\end{gathered}
$$

Taking $y=u_{n+1}$ in (3.15) and $y=u_{n}$ in (3.16), we have

$$
F\left(u_{n}, u_{n+1}\right)+\frac{1}{r_{n}}\left\langle u_{n+1}-u_{n}, u_{n}-x_{n}\right\rangle \geq 0
$$

and hence

$$
F\left(u_{n+1}, u_{n}\right)+\frac{1}{r_{n+1}}\left\langle u_{n}-u_{n+1}, u_{n+1}-x_{n+1}\right\rangle \geq 0
$$

So, from (A2) we have

$$
\left\langle u_{n+1}-u_{n}, \frac{u_{n}-x_{n}}{r_{n}}-\frac{u_{n+1}-x_{n+1}}{r_{n+1}}\right\rangle \geq 0
$$


and hence

$$
\left\langle u_{n+1}-u_{n}, u_{n}-u_{n+1}+u_{n+1}-x_{n}-\frac{r_{n}}{r_{n+1}}\left(u_{n+1}-x_{n+1}\right)\right\rangle \geq 0
$$

Without loss of generality, let us assume that there exists a real number $c$ such that $r_{n}>c>0$ for all $n \in \mathbb{N}$. Then, we have

$$
\begin{aligned}
\left\|u_{n+1}-u_{n}\right\|^{2} & \leq\left\langle u_{n+1}-u_{n}, x_{n+1}-x_{n}+\left(1-\frac{r_{n}}{r_{n+1}}\right)\left(u_{n+1}-x_{n+1}\right)\right\rangle \\
& \leq\left\|u_{n+1}-u_{n}\right\|\left\{\left\|x_{n+1}-x_{n}\right\|+\left|1-\frac{r_{n}}{r_{n+1}}\right|\left\|u_{n+1}-x_{n+1}\right\|\right\},
\end{aligned}
$$

and hence

$$
\begin{aligned}
\left\|u_{n+1}-u_{n}\right\| & \leq\left\|x_{n+1}-x_{n}\right\|+\frac{1}{r_{n+1}}\left|r_{n+1}-r_{n}\right||| u_{n+1}-x_{n+1} \| \\
& \leq\left\|x_{n+1}-x_{n}\right\|+\frac{M_{1}}{c}\left|r_{n+1}-r_{n}\right|
\end{aligned}
$$

where $M_{1}=\sup \left\{\left\|u_{n}-x_{n}\right\|: n \in \mathbb{N}\right\}$.

Notice from Lemma 2.12 that

$$
\begin{aligned}
\left\|y_{n+1}-y_{n}\right\|= & \left\|\Upsilon_{n+1}^{N} u_{n+1}-\Upsilon_{n}^{N} u_{n}\right\| \\
\leq & \left\|\left(u_{n+1}-\lambda_{k, n+1} G_{k} \Upsilon_{n+1}^{k} u_{n+1}\right)-\left(u_{n}-\lambda_{k, n} G_{k} \Upsilon_{n}^{k} u_{n}\right)\right\| \\
& +\left\|J_{M_{k}}, \lambda_{k, n+1}\left(u_{n}-\lambda_{k, n} G_{k} \Upsilon_{n}^{k} u_{n}\right)-J_{M_{k}}, \lambda_{k, n}\left(u_{n}-\lambda_{k, n} G_{k} \Upsilon_{n}^{k} u_{n}\right)\right\| \\
\leq & \left\|u_{n+1}-u_{n}\right\|+\left|\lambda_{k, n+1}-\lambda_{k, n}\right|\left\|G_{k} \Upsilon_{n}^{k} u_{n}\right\| \\
& +\frac{\left|\lambda_{k, n+1}-\lambda_{k, n}\right|}{\lambda_{k, n+1}}\left\|J_{M_{k}, \lambda_{k, n+1}}\left(u_{n}-\lambda_{k, n} G_{k} \Upsilon_{n}^{k} u_{n}\right)-\left(u_{n}-\lambda_{k, n} G_{k} \Upsilon_{n}^{k} u_{n}\right)\right\| \\
\leq & \left\|u_{n+1}-u_{n}\right\|+2 M_{2}\left|\lambda_{k, n+1}-\lambda_{k, n}\right| \\
\leq & \left\|x_{n+1}-x_{n}\right\|+\frac{M_{1}}{c}\left|r_{n+1}-r_{n}\right|+2 M_{2}\left|\lambda_{k, n+1}-\lambda_{k, n}\right|,
\end{aligned}
$$

where $M_{2}$ is an appropriate constant such that

$$
M_{2}=\max \left\{\sup _{n \geq 1}\left\{\left\|G_{k} \Upsilon_{n}^{k} u_{n}\right\|\right\}, \sup _{n \geq 1}\left\{\frac{\left\|J_{M_{k}}, \lambda_{k, n+1}\left(u_{n}-\lambda_{k, n} G_{k} \Upsilon_{n}^{k} u_{n}\right)-\left(u_{n}-\lambda_{k, n} G_{k} \Upsilon_{n}^{k} u_{n}\right)\right\|}{J_{M_{k}}, \lambda_{k, n+1}}\right\}\right\} .
$$


Journal of Applied Mathematics

Since $I-\xi_{n} B$ is nonexpansive mappings, we have the following estimates:

$$
\begin{aligned}
\left\|v_{n+1}-v_{n}\right\| & \leq\left\|P_{C}\left(y_{n+1}-\xi_{n+1} B y_{n+1}\right)-P_{C}\left(y_{n}-\xi_{n} B y_{n}\right)\right\| \\
& \leq\left\|\left(y_{n+1}-\xi_{n+1} B y_{n+1}\right)-\left(y_{n}-\xi_{n} B y_{n}\right)\right\| \\
& =\left\|\left(y_{n+1}-\xi_{n+1} B y_{n+1}\right)-\left(y_{n}-\xi_{n+1} B y_{n}\right)+\left(\xi_{n}-\xi_{n+1}\right) B y_{n}\right\| \\
& \leq\left\|\left(y_{n+1}-\xi_{n+1} B y_{n+1}\right)-\left(y_{n}-\xi_{n+1} B y_{n}\right)\right\|+\left|\xi_{n}-\xi_{n+1}\right|\left\|B y_{n}\right\| \\
& =\left\|\left(I-\xi_{n+1} B\right) y_{n+1}-\left(I-\xi_{n+1} B\right) y_{n}\right\|+\left|\xi_{n}-\xi_{n+1}\right|\left\|B y_{n}\right\| \\
& \leq\left\|y_{n+1}-y_{n}\right\|+\left|\xi_{n}-\xi_{n+1}\right|\left\|B y_{n}\right\| .
\end{aligned}
$$

Substituting (3.23) into (3.25), we obtain

$$
\begin{aligned}
\left\|v_{n+1}-v_{n}\right\| \leq & \left\|x_{n+1}-x_{n}\right\|+\frac{M_{1}}{c}\left|r_{n+1}-r_{n}\right|+2 M_{2}\left|\lambda_{k, n+1}-\lambda_{k, n}\right| \\
& +\left|\xi_{n}-\xi_{n+1}\right|\left\|B y_{n}\right\| .
\end{aligned}
$$

Indeed, define $x_{n+1}=\left(1-\beta_{n}\right) z_{n}+\beta_{n} x_{n}$ for all $n \in \mathbb{N}$. It follows that

$$
z_{n}=\frac{x_{n+1}-\beta_{n} x_{n}}{1-\beta_{n}}=\frac{\alpha_{n} f\left(x_{n}\right)+\gamma_{n} S_{n} v_{n}}{1-\beta_{n}} .
$$

Thus, we have

$$
\begin{aligned}
\left\|z_{n+1}-z_{n}\right\|= & \left\|\frac{\alpha_{n+1} f\left(x_{n+1}\right)+\gamma_{n+1} S_{n+1} v_{n+1}}{1-\beta_{n+1}}-\frac{\alpha_{n} f\left(x_{n}\right)+\gamma_{n} S_{n} v_{n}}{1-\beta_{n}}\right\| \\
= & \| \frac{\alpha_{n+1}}{1-\beta_{n+1}}\left(f\left(x_{n+1}\right)-f\left(x_{n}\right)\right)+\frac{\gamma_{n+1}}{1-\beta_{n+1}}\left(S_{n+1} v_{n+1}-S_{n} v_{n}\right) \\
& +\left(\frac{\alpha_{n+1}}{1-\beta_{n+1}}-\frac{\alpha_{n}}{1-\beta_{n}}\right) f\left(x_{n}\right)+\left(\frac{\gamma_{n+1}}{1-\beta_{n+1}}-\frac{\gamma_{n}}{1-\beta_{n}}\right) S_{n} v_{n} \| \\
\leq & \frac{\alpha_{n+1}}{1-\beta_{n+1}}\left\|f\left(x_{n+1}\right)-f\left(x_{n}\right)\right\|+\frac{\gamma_{n+1}}{1-\beta_{n+1}}\left\|S_{n+1} v_{n+1}-S_{n} v_{n}\right\| \\
& +\left|\frac{\alpha_{n+1}}{1-\beta_{n+1}}-\frac{\alpha_{n}}{1-\beta_{n}}\right|\left\|f\left(x_{n}\right)-S_{n} v_{n}\right\| \\
\leq & \frac{\psi \alpha_{n+1}}{1-\beta_{n+1}}\left\|x_{n+1}-x_{n}\right\|+\frac{\gamma_{n+1}}{1-\beta_{n+1}}\left\|S_{n+1} v_{n+1}-S_{n} v_{n}\right\| \\
& +\left|\frac{\alpha_{n+1}}{1-\beta_{n+1}}-\frac{\alpha_{n}}{1-\beta_{n}}\right|\left\|f\left(x_{n}\right)-S_{n} v_{n}\right\| .
\end{aligned}
$$

Now, compute

$$
\begin{aligned}
\left\|S_{n+1} v_{n+1}-S_{n} v_{n}\right\| \leq & \left\|S_{n+1} v_{n+1}-S_{n+1} v_{n}\right\|+\left\|S_{n+1} v_{n}-S_{n} v_{n}\right\| \\
\leq & \left\|v_{n+1}-v_{n}\right\|+\left\|S_{n+1} v_{n}-S_{n} v_{n}\right\| \\
\leq & \left\|x_{n+1}-x_{n}\right\|+\frac{M_{1}}{c}\left|r_{n+1}-r_{n}\right|+\left|\xi_{n}-\xi_{n+1}\right|\left\|B y_{n}\right\| \\
& +2 M_{2}\left|\lambda_{k, n+1}-\lambda_{k, n}\right|+\left\|S_{n+1} v_{n}-S_{n} v_{n}\right\| .
\end{aligned}
$$


Combining (3.28) and (3.29), we have

$$
\begin{aligned}
\left\|z_{n+1}-z_{n}\right\| \leq & \frac{\psi \alpha_{n+1}}{1-\beta_{n+1}}\left\|x_{n+1}-x_{n}\right\|+\frac{\gamma_{n+1}}{1-\beta_{n+1}}\left\{\left\|x_{n+1}-x_{n}\right\|+\frac{M_{1}}{c}\left|r_{n+1}-r_{n}\right|+\left|\xi_{n}-\xi_{n+1}\right|\left\|B y_{n}\right\|\right. \\
& \left.+2 M_{2}\left|\lambda_{k, n+1}-\lambda_{k, n}\right|+\left\|S_{n+1} v_{n}-S_{n} v_{n}\right\|\right\} \\
& +\left|\frac{\alpha_{n+1}}{1-\beta_{n+1}}-\frac{\alpha_{n}}{1-\beta_{n}}\right|\left\|f\left(x_{n}\right)-S_{n} v_{n}\right\| \\
\leq & \left\|x_{n+1}-x_{n}\right\|+\frac{\gamma_{n+1}}{1-\beta_{n+1}}\left\{\frac{M_{1}}{c}\left|r_{n+1}-r_{n}\right|+\left|\xi_{n}-\xi_{n+1}\right|\left\|B y_{n}\right\|\right. \\
& +\left|\frac{\alpha_{n+1}}{1-\beta_{n+1}}-\frac{\alpha_{n}}{1-\beta_{n}}\right|\left\|f\left(x_{n}\right)-S_{n} v_{n}\right\| .
\end{aligned}
$$

It follows that

$$
\begin{aligned}
\| z_{n+1}- & z_{n}\|-\| x_{n+1}-x_{n} \| \\
\leq & \frac{\gamma_{n+1}}{1-\beta_{n+1}}\left\{\frac{M_{1}}{c}\left|r_{n+1}-r_{n}\right|+\left|\xi_{n}-\xi_{n+1}\right|\left\|B y_{n}\right\|+2 M_{2}\left|\lambda_{k, n+1}-\lambda_{k, n}\right|\right\} \\
& +\frac{\gamma_{n+1}}{1-\beta_{n+1}}\left\|S_{n+1} v_{n}-S_{n} v_{n}\right\|+\left|\frac{\alpha_{n+1}}{1-\beta_{n+1}}-\frac{\alpha_{n}}{1-\beta_{n}}\right|\left\|f\left(x_{n}\right)-S_{n} v_{n}\right\| \\
\leq & \frac{\gamma_{n+1}}{1-\beta_{n+1}}\left\{\frac{M_{1}}{c}\left|r_{n+1}-r_{n}\right|+\left|\xi_{n}-\xi_{n+1}\right|\left\|B y_{n}\right\|+2 M_{2}\left|\lambda_{k, n+1}-\lambda_{k, n}\right|\right\} \\
& +\frac{\gamma_{n+1}}{1-\beta_{n+1}} \sup \left\{\left\|S_{n+1} z-S_{n} z\right\|: z \in\left\{v_{n}\right\}\right\}+\left|\frac{\alpha_{n+1}}{1-\beta_{n+1}}-\frac{\alpha_{n}}{1-\beta_{n}}\right|\left\|f\left(x_{n}\right)-S_{n} v_{n}\right\| .
\end{aligned}
$$

This together with conditions (C1)-(C6) and $\lim _{n \rightarrow \infty} \sup \left\{\left\|S_{n+1} z-S_{n} z\right\|: z \in\left\{v_{n}\right\}\right\}=0$ implies that

$$
\limsup _{n \rightarrow \infty}\left(\left\|z_{n+1}-z_{n}\right\|-\left\|x_{n+1}-x_{n}\right\|\right) \leq 0
$$

Hence, by Lemma 2.6, we obtain $\left\|z_{n}-x_{n}\right\| \rightarrow 0$ as $n \rightarrow \infty$. It then follows that

$$
\lim _{n \rightarrow \infty}\left\|x_{n+1}-x_{n}\right\|=\lim _{n \rightarrow \infty}\left(1-\beta_{n}\right)\left\|z_{n}-x_{n}\right\|=0 .
$$

By (3.26), we also have

$$
\lim _{n \rightarrow \infty}\left\|v_{n+1}-v_{n}\right\|=0
$$


Step 3. We claim that $\lim _{n \rightarrow \infty}\left\|S v_{n}-v_{n}\right\|=0$.

Since $\left\{G_{k}: k=1,2,3, \ldots, N\right\}$ is $\beta$-inverse strongly monotone mappings, by the choice of $\left\{\lambda_{k, n}\right\}$ for given $\tilde{x} \in \Omega$ and $k \in\{0,1,2, \ldots, N-1\}$, we also have

$$
\begin{aligned}
& \left\|\Upsilon_{n}^{k+1} u_{n}-\tilde{x}\right\|^{2} \\
& \quad=\left\|J_{M_{k+1}, \lambda_{k+1, n}}\left(I-\lambda_{k+1, n} G_{k+1}\right) \Upsilon_{n}^{k} u_{n}-J_{M_{k+1}, \lambda_{k+1, n}}\left(I-\lambda_{k+1, n} G_{k+1}\right) \tilde{x}\right\|^{2} \\
& \quad \leq\left\|\left(I-\lambda_{k+1, n} G_{k+1}\right) \Upsilon_{n}^{k} u_{n}-\left(I-\lambda_{k+1, n} G_{k+1}\right) \tilde{x}\right\|^{2} \\
& \quad=\left\|\left(\Upsilon_{n}^{k} u_{n}-\lambda_{k+1, n} G_{k+1} \Upsilon_{n}^{k} u_{n}\right)-\left(\tilde{x}-\lambda_{k+1, n} G_{k+1} \tilde{x}\right)\right\|^{2} \\
& \quad=\left\|\left(\Upsilon_{n}^{k} u_{n}-\tilde{x}\right)-\lambda_{k+1, n}\left(G_{k+1} \Upsilon_{n}^{k} u_{n}-G_{k+1} \tilde{x}\right)\right\|^{2} \\
& \quad=\left\|\Upsilon_{n}^{k} u_{n}-\tilde{x}\right\|^{2}-2 \lambda_{k+1, n}\left\langle\Upsilon_{n}^{k} u_{n}-\tilde{x}, G_{k+1} \Upsilon_{n}^{k} u_{n}-G_{k+1} \tilde{x}\right\rangle+\lambda_{k+1, n}^{2}\left\|G_{k+1} \Upsilon_{n}^{k} u_{n}-G_{k+1} \tilde{x}\right\|^{2} \\
& \quad \leq\left\|\Upsilon_{n}^{k} u_{n}-\tilde{x}\right\|^{2}-2 \lambda_{k+1, n} \beta\left\|G_{k+1} \Upsilon_{n}^{k} u_{n}-G_{k+1} \tilde{x}\right\|+\lambda_{k+1, n}^{2}\left\|G_{k+1} \Upsilon_{n}^{k} u_{n}-G_{k+1} \tilde{x}\right\|^{2} \\
& \quad \leq\left\|u_{n}-\tilde{x}\right\|^{2}-2 \lambda_{k+1, n} \beta\left\|G_{k+1} \Upsilon_{n}^{k} u_{n}-G_{k+1} \tilde{x}\right\|+\lambda_{k+1, n}^{2}\left\|G_{k+1} \Upsilon_{n}^{k} u_{n}-G_{k+1} \tilde{x}\right\|^{2} \\
& \quad \leq\left\|x_{n}-\tilde{x}\right\|^{2}+\lambda_{k+1, n}\left(\lambda_{k+1, n}-2 \beta\right)\left\|G_{k+1} \Upsilon_{n}^{k} u_{n}-G_{k+1} \tilde{x}\right\|^{2} .
\end{aligned}
$$

Form (3.13), we have

$$
\begin{aligned}
\left\|x_{n+1}-\tilde{x}\right\|^{2} \leq & \alpha_{n}\left\|f\left(x_{n}\right)-\tilde{x}\right\|^{2}+\beta_{n}\left\|x_{n}-\tilde{x}\right\|^{2}+\gamma_{n}\left\|v_{n}-\tilde{x}\right\|^{2} \\
\leq & \alpha_{n}\left\|f\left(x_{n}\right)-\tilde{x}\right\|^{2}+\beta_{n}\left\|x_{n}-\tilde{x}\right\|^{2}+\gamma_{n}\left\|y_{n}-\tilde{x}\right\|^{2} \\
= & \alpha_{n}\left\|f\left(x_{n}\right)-\tilde{x}\right\|^{2}+\beta_{n}\left\|x_{n}-\tilde{x}\right\|^{2}+\gamma_{n}\left\|\Upsilon_{n}^{N} u_{n}-\tilde{x}\right\|^{2} \\
\leq & \alpha_{n}\left\|f\left(x_{n}\right)-\tilde{x}\right\|^{2}+\beta_{n}\left\|x_{n}-\tilde{x}\right\|^{2}+\gamma_{n}\left\|\Upsilon_{n}^{k+1} u_{n}-\tilde{x}\right\|^{2} \\
\leq & \alpha_{n}\left\|f\left(x_{n}\right)-\tilde{x}\right\|^{2}+\beta_{n}\left\|x_{n}-\tilde{x}\right\|^{2} \\
& +\gamma_{n}\left\{\left\|x_{n}-\tilde{x}\right\|^{2}+\lambda_{k+1, n}\left(\lambda_{k+1, n}-2 \beta\right)\left\|G_{k+1} \Upsilon_{n}^{k} u_{n}-G_{k+1} \tilde{x}\right\|^{2}\right\} \\
\leq & \alpha_{n}\left\|f\left(x_{n}\right)-\tilde{x}\right\|^{2}+\left\|x_{n}-\tilde{x}\right\|^{2}+\gamma_{n} \lambda_{k+1, n}\left(\lambda_{k+1, n}-2 \beta\right)\left\|G_{k+1} \Upsilon_{n}^{k} u_{n}-G_{k+1} \tilde{x}\right\|^{2} .
\end{aligned}
$$

It follows that

$$
\begin{aligned}
& \gamma_{n} \lambda_{k+1, n}\left(2 \beta-\lambda_{k+1, n}\right)\left\|G_{k+1} \Upsilon_{n}^{k} u_{n}-G_{k+1} \tilde{x}\right\|^{2} \\
& \quad \leq \gamma_{n} c(2 \beta-d)\left\|G_{k+1} \Upsilon_{n}^{k} u_{n}-G_{k+1} \tilde{x}\right\|^{2} \\
& \quad \leq\left\|x_{n}-x_{n+1}\right\|\left(\left\|x_{n}-\tilde{x}\right\|+\left\|x_{n+1}-\tilde{x}\right\|\right)+\alpha_{n}\left\|f\left(x_{n}\right)-\tilde{x}\right\|^{2} .
\end{aligned}
$$


By condition (C2), (3.33), and $\lim \inf _{n \rightarrow \infty} \gamma_{n}>0$, we obtain

$$
\lim _{n \rightarrow \infty}\left\|G_{k+1} \Upsilon_{n}^{k} u_{n}-G_{k+1} \tilde{x}\right\|=0
$$

From Lemma 2.3(2) and as $I-\lambda_{k+1, n} G_{k+1}$ is nonexpansive, we have

$$
\begin{aligned}
&\left\|\Upsilon_{n}^{k+1} u_{n}-\tilde{x}\right\|^{2} \\
&=\left\|J_{M_{k+1}, \lambda_{k+1, n}}\left(I-\lambda_{k+1, n} G_{k+1}\right) \Upsilon_{n}^{k} u_{n}-J_{M_{k+1}, \lambda_{k+1, n}}\left(I-\lambda_{k+1, n} G_{k+1}\right) \tilde{x}\right\|^{2} \\
& \leq\left\langle\left(I-\lambda_{k+1, n} G_{k+1}\right) \Upsilon_{n}^{k} u_{n}-\left(I-\lambda_{k+1, n} G_{k+1}\right) \tilde{x}, \Upsilon_{n}^{k+1} u_{n}-\tilde{x}\right\rangle \\
&= \frac{1}{2}\left\{\left\|\left(I-\lambda_{k+1, n} G_{k+1}\right) \Upsilon_{n}^{k} u_{n}-\left(I-\lambda_{k+1, n} G_{k+1}\right) \tilde{x}\right\|^{2}+\left\|\Upsilon_{n}^{k+1} u_{n}-\tilde{x}\right\|^{2}\right. \\
&\left.-\left\|\left(I-\lambda_{k+1, n} G_{k+1}\right) \Upsilon_{n}^{k} u_{n}-\left(I-\lambda_{k+1, n} G_{k+1}\right) \tilde{x}-\left(\Upsilon_{n}^{k+1} u_{n}-\tilde{x}\right)\right\|^{2}\right\} \\
& \leq \frac{1}{2}\left\{\left\|\Upsilon_{n}^{k} u_{n}-\tilde{x}\right\|^{2}+\left\|\Upsilon_{n}^{k+1} u_{n}-\tilde{x}\right\|^{2}-\left\|\left(\Upsilon_{n}^{k} u_{n}-\Upsilon_{n}^{k+1} u_{n}\right)-\lambda_{k+1, n}\left(G_{k+1} \Upsilon_{n}^{k} u_{n}-G_{k+1} \tilde{x}\right)\right\|^{2}\right\} \\
& \leq \frac{1}{2}\left\{\left\|\Upsilon_{n}^{k} u_{n}-\tilde{x}\right\|^{2}+\left\|\Upsilon_{n}^{k+1} u_{n}-\tilde{x}\right\|^{2}-\left\|\Upsilon_{n}^{k} u_{n}-\Upsilon_{n}^{k+1} u_{n}\right\|^{2}\right. \\
&\left.-\lambda_{k+1, n}^{2}\left\|G_{k+1} \Upsilon_{n}^{k} u_{n}-G_{k+1} \tilde{x}\right\|^{2}+2 \lambda_{k+1, n}\left\langle\Upsilon_{n}^{k} u_{n}-\Upsilon_{n}^{k+1} u_{n}, G_{k+1} \Upsilon_{n}^{k} u_{n}-G_{k+1} \tilde{x}\right\rangle\right\},
\end{aligned}
$$

which yields that

$$
\begin{aligned}
& \left\|\Upsilon_{n}^{k+1} u_{n}-\tilde{x}\right\|^{2} \\
& \quad \leq\left\|\Upsilon_{n}^{k} u_{n}-\tilde{x}\right\|^{2}-\left\|\Upsilon_{n}^{k} u_{n}-\Upsilon_{n}^{k+1} u_{n}\right\|^{2}+2 \lambda_{k+1, n}\left\|\Upsilon_{n}^{k} u_{n}-\Upsilon_{n}^{k+1} u_{n}\right\|\left\|G_{k+1} \Upsilon_{n}^{k} u_{n}-G_{k+1} \tilde{x}\right\| \\
& \quad \leq\left\|u_{n}-\tilde{x}\right\|^{2}-\left\|\Upsilon_{n}^{k} u_{n}-\Upsilon_{n}^{k+1} u_{n}\right\|^{2}+2 \lambda_{k+1, n}\left\|\Upsilon_{n}^{k} u_{n}-\Upsilon_{n}^{k+1} u_{n}\right\|\left\|G_{k+1} \Upsilon_{n}^{k} u_{n}-G_{k+1} \tilde{x}\right\| \\
& \quad \leq\left\|x_{n}-\tilde{x}\right\|^{2}-\left\|\Upsilon_{n}^{k} u_{n}-\Upsilon_{n}^{k+1} u_{n}\right\|^{2}+2 \lambda_{k+1, n}\left\|\Upsilon_{n}^{k} u_{n}-\Upsilon_{n}^{k+1} u_{n}\right\|\left\|G_{k+1} \Upsilon_{n}^{k} u_{n}-G_{k+1} \tilde{x}\right\| .
\end{aligned}
$$

Substituting (3.40) into (3.36), we obtain

$$
\begin{aligned}
\left\|x_{n+1}-\tilde{x}\right\|^{2} \leq & \alpha_{n}\left\|f\left(x_{n}\right)-\tilde{x}\right\|^{2}+\beta_{n}\left\|x_{n}-\tilde{x}\right\|^{2}+\gamma_{n}\left\|\Upsilon_{n}^{k+1} u_{n}-\tilde{x}\right\|^{2} \\
\leq & \alpha_{n}\left\|f\left(x_{n}\right)-\tilde{x}\right\|^{2}+\beta_{n}\left\|x_{n}-\tilde{x}\right\|^{2}+\gamma_{n}\left\{\left\|x_{n}-\tilde{x}\right\|^{2}-\left\|\Upsilon_{n}^{k} u_{n}-\Upsilon_{n}^{k+1} u_{n}\right\|^{2}\right. \\
& \left.+2 \lambda_{k+1, n}\left\|\Upsilon_{n}^{k} u_{n}-\Upsilon_{n}^{k+1} u_{n}\right\|\left\|G_{k+1} \Upsilon_{n}^{k} u_{n}-G_{k+1} \tilde{x}\right\|\right\} \\
\leq & \alpha_{n}\left\|f\left(x_{n}\right)-\tilde{x}\right\|^{2}+\left\|x_{n}-\tilde{x}\right\|^{2}-\gamma_{n}\left\|\Upsilon_{n}^{k} u_{n}-\Upsilon_{n}^{k+1} u_{n}\right\|^{2} \\
& +2 \lambda_{k+1, n} \gamma_{n}\left\|\Upsilon_{n}^{k} u_{n}-\Upsilon_{n}^{k+1} u_{n}\right\|\left\|G_{k+1} \Upsilon_{n}^{k} u_{n}-G_{k+1} \tilde{x}\right\| .
\end{aligned}
$$


It follows that

$$
\begin{aligned}
\gamma_{n}\left\|\Upsilon_{n}^{k} u_{n}-\Upsilon_{n}^{k+1} u_{n}\right\|^{2} \leq & \left\|x_{n}-x_{n+1}\right\|\left(\left\|x_{n}-\tilde{x}\right\|+\left\|x_{n+1}-\tilde{x}\right\|\right)+\alpha_{n}\left\|f\left(x_{n}\right)-\tilde{x}\right\|^{2} \\
& +2 \lambda_{k+1, n} \Upsilon_{n}\left\|\Upsilon_{n}^{k} u_{n}-\Upsilon_{n}^{k+1} u_{n}\right\|\left\|G_{k+1} \Upsilon_{n}^{k} u_{n}-G_{k+1} \tilde{x}\right\| .
\end{aligned}
$$

By condition (C2), (3.33), (3.38), and $\liminf _{n \rightarrow \infty} \gamma_{n}>0$, we obtain

$$
\lim _{n \rightarrow \infty}\left\|\Upsilon_{n}^{k} u_{n}-\Upsilon_{n}^{k+1} u_{n}\right\|=0
$$

For $\tilde{x} \in \Omega$, we obtain

$$
\begin{aligned}
\left\|v_{n}-\tilde{x}\right\|^{2} & =\left\|P_{C}\left(y_{n}-\xi_{n} B y_{n}\right)-P_{C}\left(\tilde{x}-\xi_{n} B \tilde{x}\right)\right\|^{2} \\
& \leq\left\|\left(y_{n}-\xi_{n} B y_{n}\right)-\left(\tilde{x}-\xi_{n} B \tilde{x}\right)\right\|^{2} \\
& =\left\|\left(y_{n}-\tilde{x}\right)-\xi_{n}\left(B y_{n}-B \tilde{x}\right)\right\|^{2} \\
& \leq\left\|y_{n}-\tilde{x}\right\|^{2}-2 \xi_{n}\left\langle y_{n}-\tilde{x}, B y_{n}-B \tilde{x}\right\rangle+\xi_{n}^{2}\left\|B y_{n}-B \tilde{x}\right\|^{2} \\
& \leq\left\|y_{n}-\tilde{x}\right\|^{2}-2 \xi_{n}\left\{-\phi\left\|B y_{n}-B \tilde{x}\right\|^{2}+\omega\left\|y_{n}-\tilde{x}\right\|^{2}\right\}+\xi_{n}^{2}\left\|B y_{n}-B \tilde{x}\right\|^{2} \\
& \leq\left\|y_{n}-\tilde{x}\right\|^{2}+2 \xi_{n} \phi\left\|B y_{n}-B \tilde{x}\right\|^{2}-2 \xi_{n} \omega\left\|y_{n}-\tilde{x}\right\|^{2}+\xi_{n}^{2}\left\|B y_{n}-B \tilde{x}\right\|^{2} \\
& \leq\left\|y_{n}-\tilde{x}\right\|^{2}+2 \xi_{n} \phi\left\|B y_{n}-B \tilde{x}\right\|^{2}-\frac{2 \xi_{n} \omega}{\mu^{2}}\left\|B y_{n}-B \tilde{x}\right\|^{2}+\xi_{n}^{2}\left\|B y_{n}-B \tilde{x}\right\|^{2} \\
& \leq\left\|x_{n}-\tilde{x}\right\|^{2}+\left(2 \xi_{n} \phi+\xi_{n}^{2}-\frac{2 \xi_{n} \omega}{\mu^{2}}\right)\left\|B y_{n}-B \tilde{x}\right\|^{2} .
\end{aligned}
$$

On the other hand, we have

$$
\begin{aligned}
\left\|x_{n+1}-\tilde{x}\right\|^{2}= & \left\|\alpha_{n} f\left(x_{n}\right)+\beta_{n} x_{n}+\gamma_{n} S_{n} v_{n}-\tilde{x}\right\|^{2} \\
\leq & \alpha_{n}\left\|f\left(x_{n}\right)-\tilde{x}\right\|^{2}+\beta_{n}\left\|x_{n}-\tilde{x}\right\|^{2}+\gamma_{n}\left\|S_{n} v_{n}-\tilde{x}\right\|^{2} \\
\leq & \alpha_{n}\left\|f\left(x_{n}\right)-\tilde{x}\right\|^{2}+\beta_{n}\left\|x_{n}-\tilde{x}\right\|^{2}+\gamma_{n}\left\|v_{n}-\tilde{x}\right\|^{2} \\
\leq & \alpha_{n}\left\|f\left(x_{n}\right)-\tilde{x}\right\|^{2}+\beta_{n}\left\|x_{n}-\tilde{x}\right\|^{2} \\
& +\gamma_{n}\left\{\left\|x_{n}-\tilde{x}\right\|^{2}+\left(2 \xi_{n} \phi+\xi_{n}^{2}-\frac{2 \xi_{n} \omega}{\mu^{2}}\right)\left\|B y_{n}-B \tilde{x}\right\|^{2}\right\} \\
= & \alpha_{n}\left\|f\left(x_{n}\right)-\tilde{x}\right\|^{2}+\beta_{n}\left\|x_{n}-\tilde{x}\right\|^{2}+\gamma_{n}\left\|x_{n}-\tilde{x}\right\|^{2} \\
& +\gamma_{n}\left(2 \xi_{n} \phi+\xi_{n}^{2}-\frac{2 \xi_{n} \omega}{\mu^{2}}\right)\left\|B y_{n}-B \tilde{x}\right\|^{2} \\
\leq & \alpha_{n}\left\|f\left(x_{n}\right)-\tilde{x}\right\|^{2}+\left\|x_{n}-\tilde{x}\right\|^{2}+\gamma_{n}\left(2 \xi_{n} \phi+\xi_{n}^{2}-\frac{2 \xi_{n} \omega}{\mu^{2}}\right)\left\|B y_{n}-B \tilde{x}\right\|^{2} .
\end{aligned}
$$


It follows that

$$
\begin{aligned}
& \left(\frac{2 a \omega}{\mu^{2}}-b^{2}-2 b \phi\right) r_{n}\left\|B y_{n}-B \tilde{x}\right\|^{2} \\
& \quad \leq\left(\frac{2 \xi_{n} \omega}{\mu^{2}}-\xi_{n}^{2}-2 \xi_{n} \phi\right) r_{n}\left\|B y_{n}-B \tilde{x}\right\|^{2} \\
& \quad \leq\left\|x_{n}-\tilde{x}\right\|^{2}-\left\|x_{n+1}-\tilde{x}\right\|^{2}+\alpha_{n}\left\|f\left(x_{n}\right)-\tilde{x}\right\|^{2} \\
& \quad \leq\left\|x_{n}-x_{n+1}\right\|\left(\left\|x_{n}-\tilde{x}\right\|+\left\|x_{n+1}-\tilde{x}\right\|\right)+\alpha_{n}\left\|f\left(x_{n}\right)-\tilde{x}\right\|^{2} .
\end{aligned}
$$

It now follows from the last inequality, conditions (C2), (3.33), and $\lim \inf _{n \rightarrow \infty} \gamma_{n}>0$ that

$$
\lim _{n \rightarrow \infty}\left\|B y_{n}-B \tilde{x}\right\|=0
$$

Since $P_{C}$ is firmly nonexpansive, we have

$$
\begin{aligned}
\left\|v_{n}-\tilde{x}\right\|^{2}= & \left\|P_{C}\left(y_{n}-\xi_{n} B y_{n}\right)-P_{C}\left(\tilde{x}-\xi_{n} B \tilde{x}\right)\right\|^{2} \\
= & \left\|P_{C}\left(I-\xi_{n} B\right) y_{n}-P_{C}\left(I-\xi_{n} B\right) \tilde{x}\right\|^{2} \\
\leq & \left\langle\left(I-\xi_{n} B\right) y_{n}-\left(I-\xi_{n} B\right) \tilde{x}, v_{n}-\tilde{x}\right\rangle \\
= & \frac{1}{2}\left\{\left\|\left(I-\alpha_{n} B\right) y_{n}-\left(I-\xi_{n} B\right) \tilde{x}\right\|^{2}+\left\|v_{n}-\tilde{x}\right\|^{2}\right. \\
& \left.\quad\left\|\left(I-\xi_{n} B\right) y_{n}-\left(I-\xi_{n} B\right) \tilde{x}-\left(v_{n}-\tilde{x}\right)\right\|^{2}\right\} \\
\leq & \frac{1}{2}\left\{\left\|y_{n}-\tilde{x}\right\|^{2}+\left\|v_{n}-\tilde{x}\right\|^{2}-\left\|\left(y_{n}-v_{n}\right)-\xi_{n}\left(B y_{n}-B \tilde{x}\right)\right\|^{2}\right\} \\
\leq & \frac{1}{2}\left\{\left\|y_{n}-\tilde{x}\right\|^{2}+\left\|v_{n}-\tilde{x}\right\|^{2}-\left\|y_{n}-v_{n}\right\|^{2}\right. \\
& \left.\quad-\xi_{n}^{2}\left\|B y_{n}-B \tilde{x}\right\|^{2}+2 \xi_{n}\left\langle y_{n}-v_{n}, B y_{n}-B \tilde{x}\right\rangle\right\},
\end{aligned}
$$

which yields that

$$
\begin{aligned}
\left\|v_{n}-\tilde{x}\right\|^{2} & \leq\left\|y_{n}-\tilde{x}\right\|^{2}-\left\|y_{n}-v_{n}\right\|^{2}+2 \xi_{n}\left\|y_{n}-v_{n}\right\|\left\|B y_{n}-B \tilde{x}\right\| \\
& \leq\left\|x_{n}-\tilde{x}\right\|^{2}-\left\|y_{n}-v_{n}\right\|^{2}+2 \xi_{n}\left\|y_{n}-v_{n}\right\|\left\|B y_{n}-B \tilde{x}\right\| .
\end{aligned}
$$

Substituting (3.49) into (3.45), we obtain

$$
\begin{aligned}
\left\|x_{n+1}-\tilde{x}\right\|^{2} \leq & \alpha_{n}\left\|f\left(x_{n}\right)-\tilde{x}\right\|^{2}+\beta_{n}\left\|x_{n}-\tilde{x}\right\|^{2}+\gamma_{n}\left\|v_{n}-\tilde{x}\right\|^{2} \\
\leq & \alpha_{n}\left\|f\left(x_{n}\right)-\tilde{x}\right\|^{2}+\beta_{n}\left\|x_{n}-\tilde{x}\right\|^{2} \\
& +\gamma_{n}\left\{\left\|x_{n}-\tilde{x}\right\|^{2}-\left\|y_{n}-v_{n}\right\|^{2}+2 \xi_{n}\left\|y_{n}-v_{n}\right\|\left\|B y_{n}-B \tilde{x}\right\|\right\}
\end{aligned}
$$


Journal of Applied Mathematics

$$
\begin{aligned}
= & \alpha_{n}\left\|f\left(x_{n}\right)-\tilde{x}\right\|^{2}+\beta_{n}\left\|x_{n}-\tilde{x}\right\|^{2}+\gamma_{n}\left\|x_{n}-\tilde{x}\right\|^{2}-\gamma_{n}\left\|y_{n}-v_{n}\right\|^{2} \\
& +2 \gamma_{n} \xi_{n}\left\|y_{n}-v_{n}\right\|\left\|B y_{n}-B \tilde{x}\right\| \\
\leq & \alpha_{n}\left\|f\left(x_{n}\right)-\tilde{x}\right\|^{2}+\left\|x_{n}-\tilde{x}\right\|^{2}-\gamma_{n}\left\|y_{n}-v_{n}\right\|^{2}+2 \gamma_{n} \xi_{n}\left\|y_{n}-v_{n}\right\|\left\|B y_{n}-B \tilde{x}\right\| .
\end{aligned}
$$

It follows that

$$
\begin{aligned}
\gamma_{n}\left\|y_{n}-v_{n}\right\|^{2} \leq & \left\|x_{n}-x_{n+1}\right\|\left(\left\|x_{n}-\tilde{x}\right\|+\left\|x_{n+1}-\tilde{x}\right\|\right)+\alpha_{n}\left\|f\left(x_{n}\right)-\tilde{x}\right\|^{2} \\
& +2 \gamma_{n} \xi_{n}\left\|y_{n}-v_{n}\right\|\left\|B y_{n}-B \tilde{x}\right\| .
\end{aligned}
$$

By condition (C2), (3.33), (3.47), and $\liminf _{n \rightarrow \infty} \gamma_{n}>0$, we obtain

$$
\lim _{n \rightarrow \infty}\left\|y_{n}-v_{n}\right\|=0
$$

On the other hand, in the light of Lemma 2.11(ii) $T_{r_{n}}$ is firmly nonexpansive; so we have

$$
\begin{aligned}
\left\|u_{n}-\tilde{x}\right\|^{2} & =\left\|T_{r_{n}} x_{n}-T_{r_{n}} \tilde{x}\right\|^{2} \\
& \leq\left\langle T_{r_{n}} x_{n}-T_{r_{n}} \tilde{x}, x_{n}-\tilde{x}\right\rangle=\left\langle u_{n}-\tilde{x}, x_{n}-\tilde{x}\right\rangle \\
& =\frac{1}{2}\left(\left\|u_{n}-\tilde{x}\right\|^{2}+\left\|x_{n}-\tilde{x}\right\|^{2}-\left\|x_{n}-u_{n}\right\|^{2}\right),
\end{aligned}
$$

which implies that

$$
\left\|u_{n}-\tilde{x}\right\|^{2} \leq\left\|x_{n}-\tilde{x}\right\|^{2}-\left\|x_{n}-u_{n}\right\|^{2}
$$

Form (3.45), we have

$$
\begin{aligned}
\left\|x_{n+1}-\tilde{x}\right\|^{2} & \leq \alpha_{n}\left\|f\left(x_{n}\right)-\tilde{x}\right\|^{2}+\beta_{n}\left\|x_{n}-\tilde{x}\right\|^{2}+\gamma_{n}\left\|v_{n}-\tilde{x}\right\|^{2} \\
& \leq \alpha_{n}\left\|f\left(x_{n}\right)-\tilde{x}\right\|^{2}+\beta_{n}\left\|x_{n}-\tilde{x}\right\|^{2}+\gamma_{n}\left\|y_{n}-\tilde{x}\right\|^{2} \\
& \leq \alpha_{n}\left\|f\left(x_{n}\right)-\tilde{x}\right\|^{2}+\beta_{n}\left\|x_{n}-\tilde{x}\right\|^{2}+\gamma_{n}\left\|u_{n}-\tilde{x}\right\|^{2} \\
& \leq \alpha_{n}\left\|f\left(x_{n}\right)-\tilde{x}\right\|^{2}+\beta_{n}\left\|x_{n}-\tilde{x}\right\|^{2}+\gamma_{n}\left\{\left\|x_{n}-\tilde{x}\right\|^{2}-\left\|x_{n}-u_{n}\right\|^{2}\right\} \\
& =\alpha_{n}\left\|f\left(x_{n}\right)-\tilde{x}\right\|^{2}+\beta_{n}\left\|x_{n}-\tilde{x}\right\|^{2}+\gamma_{n}\left\|x_{n}-\tilde{x}\right\|^{2}-\gamma_{n}\left\|x_{n}-u_{n}\right\|^{2} \\
& \leq \alpha_{n}\left\|f\left(x_{n}\right)-\tilde{x}\right\|^{2}+\left\|x_{n}-\tilde{x}\right\|^{2}-\gamma_{n}\left\|x_{n}-u_{n}\right\|^{2} .
\end{aligned}
$$

It follows that

$$
\gamma_{n}\left\|x_{n}-u_{n}\right\|^{2} \leq\left\|x_{n}-x_{n+1}\right\|\left(\left\|x_{n}-\tilde{x}\right\|+\left\|x_{n+1}-\tilde{x}\right\|\right)+\alpha_{n}\left\|f\left(x_{n}\right)-\tilde{x}\right\|^{2} .
$$

By condition (C2), (3.33), and $\lim \inf _{n \rightarrow \infty} \gamma_{n}>0$, we obtain

$$
\lim _{n \rightarrow \infty}\left\|x_{n}-u_{n}\right\|=0
$$


Observe that

$$
x_{n+1}-x_{n}=\alpha_{n}\left(f\left(x_{n}\right)-x_{n}\right)+\gamma_{n}\left(S_{n} v_{n}-x_{n}\right) .
$$

By condition (C2) and (3.33), we have

$$
\lim _{n \rightarrow \infty} \gamma_{n}\left\|S_{n} v_{n}-x_{n}\right\|=\lim _{n \rightarrow \infty}\left(\left\|x_{n+1}-x_{n}\right\|-\alpha_{n}\left\|f\left(x_{n}\right)-x_{n}\right\|\right)=0 .
$$

Since

$$
\left\|S v_{n}-u_{n}\right\| \leq\left\|S v_{n}-x_{n}\right\|+\left\|x_{n}-u_{n}\right\|
$$

from (3.57) and (3.59), we have

$$
\lim _{n \rightarrow \infty}\left\|S_{n} v_{n}-u_{n}\right\|=0
$$

Form (3.55), we have

$$
\begin{aligned}
& \left\|x_{n+1}-\tilde{x}\right\|^{2} \\
& \quad \leq \alpha_{n}\left\|f\left(x_{n}\right)-\tilde{x}\right\|^{2}+\left\|x_{n}-\tilde{x}\right\|^{2}-\gamma_{n}\left\|x_{n}-u_{n}\right\|^{2} \\
& \quad \leq \alpha_{n}\left\|f\left(x_{n}\right)-\tilde{x}\right\|^{2}+\left\|x_{n}-\tilde{x}\right\|^{2}-\gamma_{n}\left\|\left(x_{n}-y_{n}\right)+\left(y_{n}-u_{n}\right)\right\|^{2} \\
& \quad \leq \alpha_{n}\left\|f\left(x_{n}\right)-\tilde{x}\right\|^{2}+\left\|x_{n}-\tilde{x}\right\|^{2}-\gamma_{n}\left\{\left\|x_{n}-y_{n}\right\|^{2}+2\left\|x_{n}-y_{n}\right\|\left\|y_{n}-u_{n}\right\|+\left\|y_{n}-u_{n}\right\|^{2}\right\} \\
& \quad=\alpha_{n}\left\|f\left(x_{n}\right)-\tilde{x}\right\|^{2}+\left\|x_{n}-\tilde{x}\right\|^{2}-\gamma_{n}\left\|x_{n}-y_{n}\right\|^{2}-2 \gamma_{n}\left\|x_{n}-y_{n}\right\|\left\|y_{n}-u_{n}\right\|-\gamma_{n}\left\|y_{n}-u_{n}\right\|^{2} \\
& \quad \leq \alpha_{n}\left\|f\left(x_{n}\right)-\tilde{x}\right\|^{2}+\left\|x_{n}-\tilde{x}\right\|^{2}-\gamma_{n}\left\|x_{n}-y_{n}\right\|^{2} .
\end{aligned}
$$

It follows that

$$
\gamma_{n}\left\|x_{n}-y_{n}\right\|^{2} \leq\left\|x_{n}-x_{n+1}\right\|\left(\left\|x_{n}-\tilde{x}\right\|+\left\|\mathrm{x}_{n+1}-\tilde{x}\right\|\right)+\alpha_{n}\left\|f\left(x_{n}\right)-\tilde{x}\right\|^{2} .
$$

By condition (C2), (3.33), and $\liminf _{n \rightarrow \infty} \gamma_{n}>0$, we obtain

$$
\lim _{n \rightarrow \infty}\left\|x_{n}-y_{n}\right\|=0 .
$$

Since

$$
\left\|u_{n}-y_{n}\right\| \leq\left\|u_{n}-x_{n}\right\|+\left\|x_{n}-y_{n}\right\|,
$$

from (3.57) and (3.64), we have

$$
\lim _{n \rightarrow \infty}\left\|u_{n}-y_{n}\right\|=0
$$


Furthermore, by the triangular inequality we also have

$$
\left\|S_{n} v_{n}-v_{n}\right\| \leq\left\|S_{n} v_{n}-u_{n}\right\|+\left\|u_{n}-y_{n}\right\|+\left\|y_{n}-v_{n}\right\|
$$

From (3.52), (3.61), and (3.66), we have

$$
\lim _{n \rightarrow \infty}\left\|S_{n} v_{n}-v_{n}\right\|=0
$$

Applying Lemma 2.9 and (3.68), we have

$$
\begin{aligned}
\left\|S v_{n}-v_{n}\right\| & \leq\left\|S v_{n}-S_{n} v_{n}\right\|+\left\|S_{n} v_{n}-v_{n}\right\| \\
& \leq \sup \left\{\left\|S z-S_{n} z\right\|: z \in\left\{v_{n}\right\}\right\}+\left\|S_{n} v_{n}-v_{n}\right\| \longrightarrow 0 .
\end{aligned}
$$

Step 4. We claim that $\limsup _{n \rightarrow \infty}\left\langle f\left(x^{*}\right)-x^{*}, x_{n}-x^{*}\right\rangle \leq 0$.

Indeed, we choose a subsequence $\left\{v_{n_{i}}\right\}$ of $\left\{v_{n}\right\}$ such that

$$
\limsup _{n \rightarrow \infty}\left\langle f\left(x^{*}\right)-x^{*}, S v_{n}-x^{*}\right\rangle=\lim _{i \rightarrow \infty}\left\langle f\left(x^{*}\right)-x^{*}, S v_{n_{i}}-x^{*}\right\rangle .
$$

Without loss of generality, let $\left\{v_{n_{i}}\right\} \rightarrow z \in C$. From $\left\|S v_{n}-v_{n}\right\| \rightarrow 0$, we obtain $S v_{n_{i}} \rightarrow z$. Then, (3.70) reduces to

$$
\limsup _{n \rightarrow \infty}\left\langle f\left(x^{*}\right)-x^{*}, S v_{n}-x^{*}\right\rangle=\left\langle f\left(x^{*}\right)-x^{*}, z-x^{*}\right\rangle .
$$

In order to show $\left\langle\mathrm{f}\left(x^{*}\right)-x^{*}, z-x^{*}\right\rangle \leq 0$, it suffices to show that

$$
z \in \Omega: \bigcap_{n=1}^{\infty} F\left(S_{n}\right) \cap\left(\bigcap_{k=1}^{N} I\left(G_{k}, M_{k}\right)\right) \cap \operatorname{VI}(C, B) \cap \operatorname{EP}(F) .
$$

Firstly, we will show $z \in F(S)=\bigcap_{n=1}^{\infty} F\left(S_{n}\right)$.

Assume $z \notin F(S)$. By Opial's theorem (Lemma 2.5) and $\left\|S v_{n}-v_{n}\right\| \rightarrow 0$, we have

$$
\begin{aligned}
\liminf _{i \rightarrow \infty}\left\|v_{n_{i}}-z\right\| & <\liminf _{i \rightarrow \infty}\left\|v_{n_{i}}-S z\right\| \\
& =\liminf _{i \rightarrow \infty}\left\|v_{n_{i}}-S v_{n_{i}}+S v_{n_{i}}-S z\right\| \\
& =\liminf _{i \rightarrow \infty}\left\|S v_{n_{i}}-S z\right\| \\
& \leq \liminf _{i \rightarrow \infty}\left\|v_{n_{i}}-z\right\| .
\end{aligned}
$$

This is a contradiction. Thus, we obtain $z \in F(S)$. 
Next, we will show that $z \in \operatorname{VI}(C, B)$.

Let

$$
T w_{1}= \begin{cases}B w_{1}+N_{C} w_{1}, & w_{1} \in C \\ \emptyset, & w_{1} \notin C .\end{cases}
$$

Since $B$ is relaxed $(\phi, \omega)$-cocoercive, $\mu$-Lipschitz continuous with $\omega>\phi \mu^{2}$, we obtain

$$
\langle B x-B y, x-y\rangle \geq(-\phi)\|B x-B y\|^{2}+\omega\|x-y\|^{2} \geq\left(\omega-\phi \mu^{2}\right)\|x-y\|^{2} \geq 0,
$$

which yields that $B$ is monotone. Then $T$ is maximal monotone (see [23]). Let $\left(w_{1}, w_{2}\right) \in G(T)$. Since $w_{2}-B w_{1} \in N_{C}\left(w_{1}\right)$ and $v_{n} \in C$, we have $\left\langle w_{1}-v_{n}, w_{2}-B w_{1}\right\rangle \geq 0$. On the other hand, from $v_{n}=P_{C}\left(y_{n}-\xi_{n} B y_{n}\right)$, we have

$$
\left\langle w_{1}-v_{n}, v_{n}-\left(y_{n}-\xi_{n} B y_{n}\right)\right\rangle \geq 0
$$

that is,

$$
\left\langle w_{1}-v_{n}, \frac{v_{n}-y_{n}}{\xi_{n}}+B y_{n}\right\rangle \geq 0
$$

Therefore, we obtain

$$
\begin{aligned}
\left\langle w_{1}-v_{n_{i}}, w_{2}\right\rangle \geq & \left\langle w_{1}-v_{n_{i}}, B w_{1}\right\rangle \\
\geq & \left\langle w_{1}-v_{n_{i}}, B w_{1}\right\rangle-\left\langle w_{1}-v_{n_{i}}, \frac{v_{n_{i}}-y_{n_{i}}}{\xi_{n_{i}}}+B y_{n_{i}}\right\rangle \\
= & \left\langle w_{1}-v_{n_{i}}, B w_{1}-B y_{n_{i}}-\frac{v_{n_{i}}-y_{n_{i}}}{\xi_{n_{i}}}\right\rangle \\
= & \left\langle w_{1}-v_{n_{i}}, B w_{1}-B v_{n_{i}}\right\rangle+\left\langle w_{1}-v_{n_{i}}, B v_{n_{i}}-B y_{n_{i}}\right\rangle \\
& -\left\langle w_{1}-v_{n_{i}}, \frac{v_{n_{i}}-y_{n_{i}}}{\xi n_{i}}\right\rangle \\
\geq & \left\langle w_{1}-v_{n_{i}}, B v_{n_{i}}\right\rangle-\left\langle w_{1}-v_{n_{i}}, \frac{v_{n_{i}}-y_{n_{i}}}{\xi_{n_{i}}}+B y_{n_{i}}\right\rangle \\
= & \left\langle w_{1}-v_{n_{i}}, B v_{n_{i}}-B y_{n_{i}}\right\rangle-\left\langle w_{1}-v_{n_{i}}, \frac{v_{n_{i}}-y_{n_{i}}}{\xi_{n_{i}}}\right\rangle .
\end{aligned}
$$

Noting that $\left\|v_{n_{i}}-y_{n_{i}}\right\| \rightarrow 0$ and $B$ is relaxed $(\phi, \omega)$-cocoercive and (3.78), we obtain

$$
\left\langle w_{1}-z, w_{2}\right\rangle \geq 0
$$

Since $T$ is maximal monotone, we have $z \in T^{-1} 0$, and hence $z \in \operatorname{VI}(C, B)$.

Now, we will show that $z \in \bigcap_{k=1}^{N} I\left(G_{k}, M_{k}\right)$. 
For this purpose, let $k \in\{1,2,3, \ldots, N\}$ and $G_{k}$ is $\beta$-inverse strongly monotone, $G_{k}$ is an $1 / \beta$-Lipschitz continuous monotone mapping. From Lemma 2.2, we know that $M_{k}+G_{k}$ is maximal monotone. Let $(v, g) \in G\left(M_{k}+G_{k}\right)$; that is, $g-G_{k} v \in M_{k}(v)$. On the other hand, since $\Upsilon_{n_{i}}^{k} u_{n_{i}}=J_{M_{k}, \lambda_{k, n_{i}}}\left(\Upsilon_{n_{i}}^{k-1} u_{n_{i}}-\lambda_{k, n_{i}} G_{k} \Upsilon_{n_{i}}^{k-1} u_{n_{i}}\right)$, we have

$$
\Upsilon_{n_{i}}^{k} u_{n_{i}}-\lambda_{k, n_{i}} G_{k} \Upsilon_{n_{i}}^{k} u_{n_{i}} \in\left(I+\lambda_{k, n_{i}} M_{k}\right)\left(\Upsilon_{n_{i}}^{k} u_{n_{i}}\right)
$$

that is,

$$
\frac{1}{\lambda_{k, n_{i}}}\left(\Upsilon_{n_{i}}^{k-1} u_{n_{i}}-\Upsilon_{n_{i}}^{k} u_{n_{i}}-\lambda_{k, n_{i}} G_{k} \Upsilon_{n_{i}}^{k-1} u_{n_{i}}\right) \in M_{k}\left(\Upsilon_{n_{i}}^{k} u_{n_{i}}\right)
$$

By virtue of the maximal monotonicity of $M_{k}+G_{k}$, we have

$$
\left\langle v-\Upsilon_{n_{i}}^{k} u_{n_{i}}, g-G_{k} v-\frac{1}{\lambda_{k, n_{i}}}\left(\Upsilon_{n_{i}}^{k-1} u_{n_{i}}-\Upsilon_{n_{i}}^{k} u_{n_{i}}-\lambda_{k, n_{i}} G_{k} \Upsilon_{n_{i}}^{k-1} u_{n_{i}}\right)\right\rangle \geq 0
$$

and so

$$
\begin{aligned}
\left\langle v-\Upsilon_{n_{i}}^{k} u_{n_{i}}, g\right\rangle \geq & \left\langle v-\Upsilon_{n_{i}}^{k} u_{n_{i}}, G_{k} v+\frac{1}{\lambda_{k, n_{i}}}\left(\Upsilon_{n_{i}}^{k-1} u_{n_{i}}-\Upsilon_{n_{i}}^{k} u_{n_{i}}-\lambda_{k, n_{i}} G_{k} \Upsilon_{n_{i}}^{k-1} u_{n_{i}}\right)\right\rangle \\
= & \left\langle v-\Upsilon_{n_{i}}^{k} u_{n_{i}}, G_{k} v-G_{k} \Upsilon_{n_{i}}^{k} u_{n_{i}}+G_{k} \Upsilon_{n_{i}}^{k} u_{n_{i}}-G_{k} \Upsilon_{n_{i}}^{k-1} u_{n_{i}}\right. \\
& \left.+\frac{1}{\lambda_{k, n_{i}}}\left(\Upsilon_{n_{i}}^{k-1} u_{n_{i}}-\Upsilon_{n_{i}}^{k} u_{n_{i}}\right)\right\rangle \\
\geq & 0+\left\langle v-\Upsilon_{n_{i}}^{k} u_{n_{i}}, G_{k} \Upsilon_{n_{i}}^{k} u_{n_{i}}-G_{k} \Upsilon_{n_{i}}^{k-1} u_{n_{i}}\right\rangle \\
& +\left\langle v-\Upsilon_{n_{i}}^{k} u_{n_{i}}, \frac{1}{\lambda_{k, n_{i}}}\left(\Upsilon_{n_{i}}^{k-1} u_{n_{i}}-\Upsilon_{n_{i}}^{k} u_{n_{i}}\right)\right\rangle .
\end{aligned}
$$

From $\left\|\Upsilon_{n}^{k} u_{n}-\Upsilon_{n}^{k+1} u_{n}\right\| \rightarrow 0$, we also obtain that $\Upsilon_{n_{i}}^{k} u_{n_{i}} \rightarrow z$ and $\left\{G_{k}: k=1,2,3, \ldots, N\right\}$ are Lipschitz continuous; we have

$$
\lim _{n \rightarrow \infty}\left\langle v-\Upsilon_{n_{i}}^{k} u_{n_{i}}, g\right\rangle=\langle v-z, g\rangle \geq 0
$$

Since $M_{k}+G_{k}$ is maximal monotone, we have $\theta \in\left(M_{k}+G_{k}\right)(z)$; that is, $z \in \bigcap_{k=1}^{N} I\left(G_{k}, M_{k}\right)$. Finally, we will show that $z \in \operatorname{EP}(F)$.

Since $u_{n}=T_{r_{n}} x_{n}$, we have

$$
F\left(u_{n}, y\right)+\frac{1}{r_{n}}\left\langle y-u_{n}, u_{n}-x_{n}\right\rangle \geq 0, \quad \forall y \in C
$$


If follows from (A2) that

$$
\frac{1}{r_{n}}\left\langle y-u_{n}, u_{n}-x_{n}\right\rangle \geq-F\left(u_{n}, y\right) \geq F\left(y, u_{n}\right)
$$

and hence

$$
\left\langle y-u_{n_{i}}, \frac{u_{n_{i}}-x_{n_{i}}}{r_{n_{i}}}\right\rangle \geq F\left(y, u_{n_{i}}\right)
$$

Since $\left(u_{n_{i}}-x_{n_{i}}\right) / r_{n_{i}} \rightarrow 0$ and $u_{n_{i}} \rightarrow z$, it follows by (A4) that $F(y, z) \leq 0$ for all $y \in H$. For $t$ with $0<t \leq 1$ and $y \in H$, let $y_{t}=t y+(1-t) z$. Since $y \in H$ and $z \in H$, we have $y_{t} \in H$, and hence $F\left(y_{t}, z\right) \leq 0$. So, from (A1) and (A4) we have

$$
0=F\left(y_{t}, y_{t}\right) \leq t F\left(y_{t}, y\right)+(1-t) F\left(y_{t}, z\right) \leq t F\left(y_{t}, y\right)
$$

and hence $F\left(y_{t}, y\right) \geq 0$. From (A3), we have $F(z, y) \geq 0$ for all $y \in H$ and hence $z \in \operatorname{EP}(F)$. Therefore, it follows that $z \in \Omega$.

Since $x^{*}=P_{\Omega} f\left(x^{*}\right)$, we have

$$
\begin{aligned}
\limsup _{n \rightarrow \infty}\left\langle f\left(x^{*}\right)-x^{*}, x_{n}-x^{*}\right\rangle & =\limsup _{n \rightarrow \infty}\left\langle f\left(x^{*}\right)-x^{*}, S v_{n}-x^{*}\right\rangle \\
& =\lim _{i \rightarrow \infty}\left\langle f\left(x^{*}\right)-x^{*}, S v_{n_{i}}-x^{*}\right\rangle \\
& =\left\langle f\left(x^{*}\right)-x^{*}, z-x^{*}\right\rangle \leq 0 .
\end{aligned}
$$

On the other hand, we have

$$
\begin{array}{r}
\limsup _{n \rightarrow \infty}\left\langle f\left(x^{*}\right)-x^{*}, x_{n+1}-x^{*}\right\rangle \leq \\
\limsup _{n \rightarrow \infty}\left\langle f\left(x^{*}\right)-x^{*}, x_{n+1}-x_{n}\right\rangle \\
+\limsup _{n \rightarrow \infty}\left\langle f\left(x^{*}\right)-x^{*}, x_{n}-x^{*}\right\rangle .
\end{array}
$$

Since $\left\|x_{n+1}-x_{n}\right\| \rightarrow 0$ as $n \rightarrow \infty$ and (3.91), we have

$$
\limsup _{n \rightarrow \infty}\left\langle f\left(x^{*}\right)-x^{*}, x_{n+1}-x^{*}\right\rangle \leq 0
$$


Step 5. We claim that $\lim _{n \rightarrow \infty}\left\|x_{n}-x^{*}\right\|=0$.

Indeed, from (3.2) and (3.12), we obtain

$$
\begin{aligned}
\left\|x_{n+1}-x^{*}\right\|^{2}= & \left\langle\alpha_{n} f\left(x_{n}\right)+\beta_{n} x_{n}+\gamma_{n} S_{n} v_{n}-x^{*}, x_{n+1}-x^{*}\right\rangle \\
= & \alpha_{n}\left\langle f\left(x_{n}\right)-x^{*}, x_{n+1}-x^{*}\right\rangle+\beta_{n}\left\langle x_{n}-x^{*}, x_{n+1}-x^{*}\right\rangle+\gamma_{n}\left\langle S_{n} v_{n}-x^{*}, x_{n+1}-x^{*}\right\rangle \\
\leq & \frac{1}{2} \beta_{n}\left(\left\|x_{n}-x^{*}\right\|^{2}+\left\|x_{n+1}-x^{*}\right\|^{2}\right)+\frac{1}{2} \gamma_{n}\left(\left\|v_{n}-x^{*}\right\|^{2}+\left\|x_{n+1}-x^{*}\right\|^{2}\right) \\
& +\alpha_{n}\left\langle f\left(x_{n}\right)-f\left(x^{*}\right), x_{n+1}-x^{*}\right\rangle+\alpha_{n}\left\langle f\left(x^{*}\right)-x^{*}, x_{n+1}-x^{*}\right\rangle \\
\leq & \frac{1}{2}\left(1-\alpha_{n}\right)\left(\left\|x_{n}-x^{*}\right\|^{2}+\left\|x_{n+1}-x^{*}\right\|^{2}\right)+\frac{1}{2} \alpha_{n}\left(\left\|f\left(x_{n}\right)-f\left(x^{*}\right)\right\|^{2}+\left\|x_{n+1}-x^{*}\right\|^{2}\right) \\
& +\alpha_{n}\left\langle f\left(x^{*}\right)-x^{*}, x_{n+1}-x^{*}\right\rangle \\
\leq & \frac{1}{2}\left[1-\alpha_{n}\left(1-\psi^{2}\right)\right]\left\|x_{n}-x^{*}\right\|^{2}+\frac{1}{2}\left(1-\alpha_{n}\right)\left\|x_{n+1}-x^{*}\right\|^{2}+\frac{1}{2} \alpha_{n}\left\|x_{n+1}-x^{*}\right\|^{2} \\
& +\alpha_{n}\left\langle f\left(x^{*}\right)-x^{*}, x_{n+1}-x^{*}\right\rangle,
\end{aligned}
$$

which implies that

$$
\begin{aligned}
\left\|x_{n+1}-x^{*}\right\|^{2} & \leq\left[1-\alpha_{n}\left(1-\psi^{2}\right)\right]\left\|x_{n}-x^{*}\right\|^{2}+2 \alpha_{n}\left\langle f\left(x^{*}\right)-x^{*}, x_{n+1}-x^{*}\right\rangle \\
& =\left(1-b_{n}\right)\left\|x_{n}-x^{*}\right\|^{2}+\delta_{n},
\end{aligned}
$$

where $b_{n}=\alpha_{n}\left(1-\psi^{2}\right)$ and $\delta_{n}=2 \alpha_{n}\left\langle f\left(x^{*}\right)-x^{*}, x_{n+1}-x^{*}\right\rangle$. It is easy to see that $b_{n} \rightarrow 0$, $\sum_{n=1}^{\infty} b_{n}=\infty$, and $\lim \sup _{n \rightarrow \infty} \delta_{n} / b_{n} \leq 0$. Applying Lemma 2.7 to (3.93), we conclude that

$$
x_{n} \rightarrow x^{*}=P_{\Omega} f\left(x^{*}\right) .
$$

Consequently, also $\left\{y_{n}\right\}$ converges strongly to $x^{*}$. The proof is now complete.

As in [21, Theorem 4.1], we can generate a sequence $\left\{S_{n}\right\}$ of nonexpansive mappings satisfying condition $\sum_{n=1}^{\infty} \sup \left\{\left\|S_{n+1} z-S_{n} z\right\|: z \in K\right\}<\infty$ for any bounded subset $K$ of $C$ by using convex combination of general sequence $\left\{T_{k}\right\}$ of nonexpansive mappings with a common fixed point.

Corollary 3.2. Let $C$ be a nonempty closed convex subset of a real Hilbert space $H$, and let $B: C \rightarrow$ $H$ be relaxed $(\phi, \omega)$-cocoercive and $\mu$-Lipschitz continuous with $\omega>\phi \mu^{2}$, for some $\phi, \omega, \mu>0$. Let $\mathcal{G}=\left\{G_{k}: k=1,2,3, \ldots, N\right\}$ be a finite family of $\beta$-inverse strongly monotone mappings from $C$ into $H$, and let $F$ be a bifunction from $C \times C \rightarrow \mathbb{R}$ satisfying (A1)-(A4). Let $f: C \rightarrow C$ be a contraction with coefficient $\psi(0 \leq \psi<1)$, and let $\left\{\delta_{n}^{k}\right\}$ be a family of nonnegative numbers with indices $n, k \in \mathbb{N}$ with $k \leq n$ such that

$$
\Omega: F\left(\bigcap_{k=1}^{\infty} F\left(T_{k}\right)\right) \cap\left(\bigcap_{k=1}^{N} I\left(G_{k}, M_{k}\right)\right) \cap \operatorname{VI}(C, B) \cap \operatorname{EP}(F) \neq \emptyset .
$$


Let the sequences $\left\{x_{n}\right\}$ and $\left\{y_{n}\right\}$ be generated by

$$
\begin{aligned}
x_{1} & =x \in \text { C chosen arbitrarily, } \\
y_{n} & =J_{M_{N}, \lambda_{N, n}}\left(I-\lambda_{N, n} G_{n}\right) \cdots J_{M_{2}, \lambda_{2, n}}\left(I-\lambda_{2, n} G_{2}\right) J_{M_{1, \lambda}, \lambda_{1, n}}\left(I-\lambda_{1, n} G_{1}\right) T_{r_{n}} x_{n}, \\
x_{n+1} & =\alpha_{n} f\left(x_{n}\right)+\beta_{n} x_{n}+\gamma_{n} \sum_{k=1}^{n} \delta_{n}^{k} T_{k} P_{C}\left(y_{n}-\xi_{n} B y_{n}\right), \quad \forall n \geq 1,
\end{aligned}
$$

where $\left\{\alpha_{n}\right\},\left\{\beta_{n}\right\},\left\{\gamma_{n}\right\} \subset(0,1)$ and $\left\{\xi_{n}\right\},\left\{r_{n}\right\} \subset(0, \infty)$ satisfy the following conditions:

(C1) $\alpha_{n}+\beta_{n}+\gamma_{n}=1$,

(C2) $\lim _{n \rightarrow \infty} \alpha_{n}=0, \sum_{n=1}^{\infty} \alpha_{n}=\infty$,

(C3) $0<\liminf _{n \rightarrow \infty} \beta_{n} \leq \lim \sup _{n \rightarrow \infty} \beta_{n}<1$,

(C4) $\left\{\xi_{n}\right\} \subset[a, b]$ for some $a, b$ with $0 \leq a \leq b \leq 2\left(\omega-\phi \mu^{2}\right) / \mu^{2}$ and $\lim _{n \rightarrow \infty}\left|\xi_{n+1}-\xi_{n}\right|=0$,

(C5) $\left\{\lambda_{k, n}\right\}_{k=1}^{N} \subset[c, d] \subset(0,2 \beta)$ and $\lim _{n \rightarrow \infty}\left|\lambda_{k, n+1}-\lambda_{k, n}\right|=0$, for each $k \in\{1,2, \ldots, N\}$,

(C6) $\liminf _{n \rightarrow \infty} r_{n}>0$ and $\lim _{n \rightarrow \infty}\left|r_{n+1}-r_{n}\right|=0$,

(C7) $\sum_{k=1}^{n} \delta_{n}^{k}$, for all $n \in \mathbb{N}, \lim _{n \rightarrow \infty} \delta_{n}^{k}>0$, for all $k \in \mathbb{N}$ and $\sum_{n=1}^{k} \sum_{k=1}^{n}\left|\delta_{n+1}^{k}-\delta_{n}^{k}\right|<\infty$.

Then, the sequences $\left\{x_{n}\right\}$ and $\left\{y_{n}\right\}$ converge strongly to the same point $x^{*} \in \Omega$, where $x^{*}=P_{\Omega} f\left(x^{*}\right)$.

In Theorem 3.1, taking $N=1$ and $S_{n}=S$, then we have the following corollary.

Corollary 3.3. Let $C$ be a nonempty closed convex subset of a real Hilbert space $H$, and let $B: C \rightarrow$ $H$ be relaxed $(\phi, \omega)$-cocoercive and $\mu$-Lipschitz continuous with $\omega>\phi \mu^{2}$, for some $\phi, \omega, \mu>0$. Let $G$ be an $\beta$-inverse strongly monotone mappings from $C$ into $H$, and let $F$ be a bifunction from $C \times C \rightarrow \mathbb{R}$ satisfying (A1)-(A4). Let $f: C \rightarrow C$ be a contraction with coefficient $\psi(0 \leq \psi<1)$, and let $S$ be a nonexpansive mappings of $C$ into itself such that

$$
\Omega: F(S) \cap I(G, M) \cap \operatorname{VI}(C, B) \cap \operatorname{EP}(F) \neq \emptyset .
$$

Let the sequences $\left\{x_{n}\right\}$ and $\left\{y_{n}\right\}$ be generated by

$$
\begin{aligned}
x_{1} & =x \in C \text { chosen arbitrarily, } \\
F\left(u_{n}, y\right) & +\frac{1}{r_{n}}\left\langle y-u_{n}, u_{n}-x_{n}\right\rangle \geq 0, \quad \forall y \in C, \\
y_{n} & =J_{M, \lambda_{n}}\left(I-\lambda_{n} G\right) u_{n}, \\
x_{n+1} & =\alpha_{n} f\left(x_{n}\right)+\beta_{n} x_{n}+\gamma_{n} S P_{C}\left(y_{n}-\xi_{n} B y_{n}\right), \quad \forall n \geq 1,
\end{aligned}
$$

where $\left\{\alpha_{n}\right\},\left\{\beta_{n}\right\},\left\{\gamma_{n}\right\} \subset(0,1)$ and $\left\{\xi_{n}\right\},\left\{r_{n}\right\} \subset(0, \infty)$ satisfy the following conditions:

(C1) $\alpha_{n}+\beta_{n}+\gamma_{n}=1$,

(C2) $\lim _{n \rightarrow \infty} \alpha_{n}=0, \sum_{n=1}^{\infty} \alpha_{n}=\infty$,

(C3) $0<\liminf _{n \rightarrow \infty} \beta_{n} \leq \lim \sup _{n \rightarrow \infty} \beta_{n}<1$,

(C4) $\left\{\xi_{n}\right\} \subset[a, b]$ for some $a, b$ with $0 \leq a \leq b \leq 2\left(\omega-\phi \mu^{2}\right) / \mu^{2}$ and $\lim _{n \rightarrow \infty}\left|\xi_{n+1}-\xi_{n}\right|=0$, 
(C5) $\left\{\lambda_{n}\right\} \subset[c, d] \subset(0,2 \beta)$ and $\lim _{n \rightarrow \infty}\left|\lambda_{n+1}-\lambda_{n}\right|=0$,

(C6) $\liminf _{n \rightarrow \infty} r_{n}>0$ and $\lim _{n \rightarrow \infty}\left|r_{n+1}-r_{n}\right|=0$.

Then, the sequences $\left\{x_{n}\right\}$ and $\left\{y_{n}\right\}$ converge strongly to the same point $x^{*} \in \Omega$, where $x^{*}=P_{\Omega} f\left(x^{*}\right)$.

\section{Acknowledgments}

This research was supported by the Rajamangala University of Technology Rattanakosin Research and Development Institute, the Thailand Research Fund, and the Commission on Higher Education under Grant no. MRG5480206. The second author would like to thank the Higher Education Research Promotion and National Research University Project of Thailand, Office ofthe Higher Education Commission (under the Project NRU-CSEC no. 54000267) for financial support. The authors are very grateful to the referees for their careful reading, comments, and suggestions which improved the presentation of this article.

\section{References}

[1] K. Goebel and W. A. Kirk, Topics in Metric Fixed Point Theory, vol. 28 of Cambridge Studies in Advanced Mathematics, Cambridge University Press, Cambridge, Mass, USA, 1990.

[2] W. Takahashi, Nonlinear Functional Analysis, Yokohama Publishers, Yokohama, Japan, 2000.

[3] W. Takahashi and M. Toyoda, "Weak convergence theorems for nonexpansive mappings and monotone mappings," Journal of Optimization Theory and Applications, vol. 118, no. 2, pp. 417-428, 2003.

[4] S.-S. Zhang, J. H. W. Lee, and C. K. Chan, "Algorithms of common solutions to quasi variational inclusion and fixed point problems," Applied Mathematics and Mechanics English Edition, vol. 29, no. 5, pp. 571-581, 2008.

[5] E. Blum and W. Oettli, "From optimization and variational inequalities to equilibrium problems," The Mathematics Student, vol. 63, no. 1-4, pp. 123-145, 1994.

[6] S. D. Flåm and A. S. Antipin, "Equilibrium programming using proximal-like algorithms," Mathematical Programming, vol. 78, no. 1, pp. 29-41, 1997.

[7] P. Cholamjiak and S. Suantai, "A new hybrid algorithm for variational inclusions, generalized equilibrium problems, and a finite family of quasi-nonexpansive mappings," Fixed Point Theory and Applications, vol. 2009, Article ID 350979, 20 pages, 2009.

[8] P. Cholamjiak and S. Suantai, "Convergence analysis for a system of equilibrium problems and a countable family of relatively quasi-nonexpansive mappings in Banach spaces," Abstract and Applied Analysis, vol. 2010, Article ID 141376, 17 pages, 2010.

[9] P. Cholamjiak and S. Suantai, "Existence and iteration for a mixed equilibrium problem and a countable family of nonexpansive mappings in Banach spaces," Computers $\mathcal{E}$ Mathematics with Applications, vol. 61, no. 9, pp. 2725-2733, 2011.

[10] P. Cholamjiak and S. Suantai, "An iterative method for equilibrium problems and a finite family of relatively nonexpansive mappings in a Banach space," Applied Mathematics and Computation, vol. 217, no. 8, pp. 3825-3831, 2010.

[11] A. N. Iusem and M. Nasri, "Korpelevich's method for variational inequality problems in Banach spaces," Journal of Global Optimization, vol. 50, no. 1, pp. 59-76, 2011.

[12] Y. Yao, M. A. Noor, and Y. C. Liou, "Strong convergence of a modified extra-gradient method to the minimum-norm solution of variational inequalities," Abstract and Applied Analysis, vol. 2012, Article ID 817436, 9 pages, 2012.

[13] Y. Yao, R. Chen, and Y. C. Liou, "A unified implicit algorithm for solving the triple-hierarchical constrained optimization problem," Mathematical E Computer Modelling, vol. 55, pp. 1506-1515, 2012.

[14] Y. Yao, M. A. Noor, Y. C. Liou, and S. M. Kang, "Iterative algorithms for general multi-valued variational inequalities," Abstract and Applied Analysis, vol. 2012, Article ID 768272, 10 pages, 2012.

[15] Y. Su, M. Shang, and X. Qin, "An iterative method of solution for equilibrium and optimization problems," Nonlinear Analysis: Theory, Methods E Applications, vol. 69, no. 8, pp. 2709-2719, 2008. 
[16] H. Brézis, "Opérateur maximaux monotones," in Mathematics Studies, vol. 5, North-Holland, Amsterdam, The Netherlands, 1973.

[17] B. Lemaire, "Which fixed point does the iteration method select?" in Recent Advances in Optimization (Trier, 1996), vol. 452 of Lecture Notes in Economics and Mathematical Systems, pp. 154-167, Springer, Berlin, Germany, 1997.

[18] Z. Opial, "Weak convergence of the sequence of successive approximations for nonexpansive mappings," Bulletin of the American Mathematical Society, vol. 73, pp. 591-597, 1967.

[19] T. Suzuki, "Strong convergence of Krasnoselskii and Mann's type sequences for one-parameter nonexpansive semigroups without Bochner integrals," Journal of Mathematical Analysis and Applications, vol. 305, no. 1, pp. 227-239, 2005.

[20] H.-K. Xu, "Viscosity approximation methods for nonexpansive mappings," Journal of Mathematical Analysis and Applications, vol. 298, no. 1, pp. 279-291, 2004.

[21] K. Aoyama, Y. Kimura, W. Takahashi, and M. Toyoda, "Approximation of common fixed points of a countable family of nonexpansive mappings in a Banach space," Nonlinear Analysis: Theory, Methods $\mathcal{E}$ Applications, vol. 67, no. 8, pp. 2350-2360, 2007.

[22] S. Takahashi, W. Takahashi, and M. Toyoda, "Strong convergence theorems for maximal monotone operators with nonlinear mappings in Hilbert spaces," Journal of Optimization Theory and Applications, vol. 147, no. 1, pp. 27-41, 2010.

[23] R. T. Rockafellar, "On the maximality of sums of nonlinear monotone operators," Transactions of the American Mathematical Society, vol. 149, pp. 75-88, 1970. 


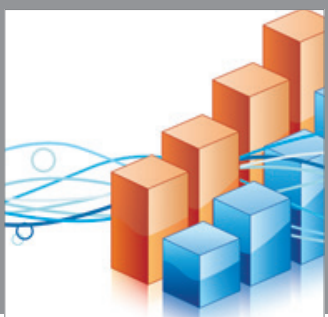

Advances in

Operations Research

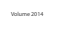

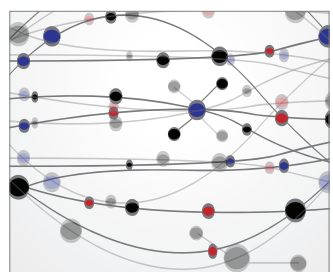

\section{The Scientific} World Journal
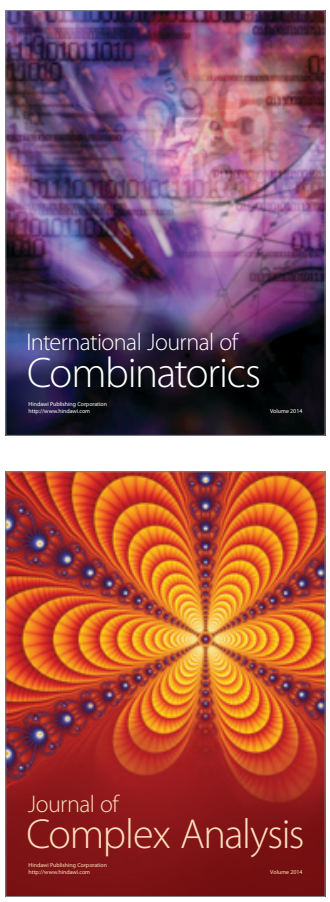

International Journal of

Mathematics and

Mathematical

Sciences
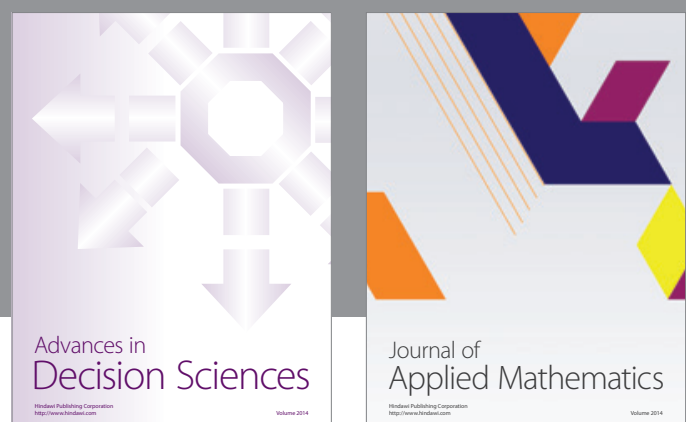

Journal of

Applied Mathematics
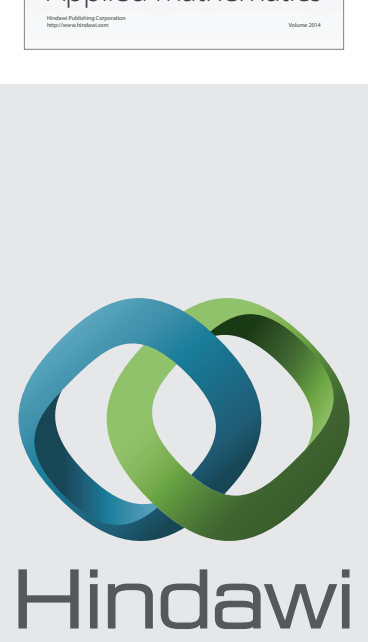

Submit your manuscripts at http://www.hindawi.com
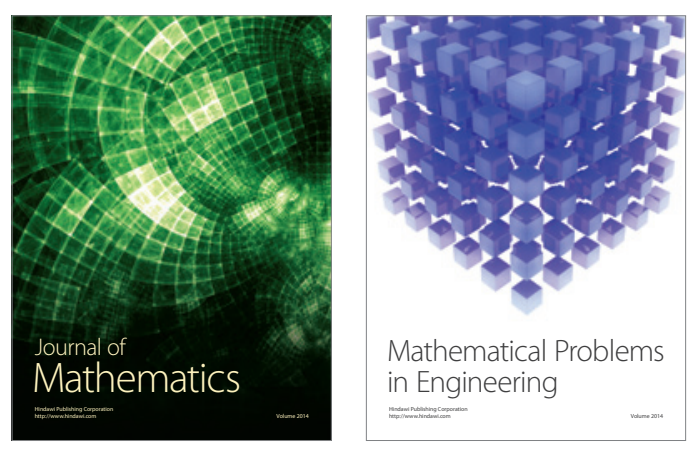

Mathematical Problems in Engineering
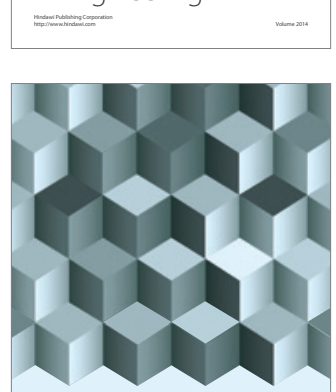

Journal of

Function Spaces
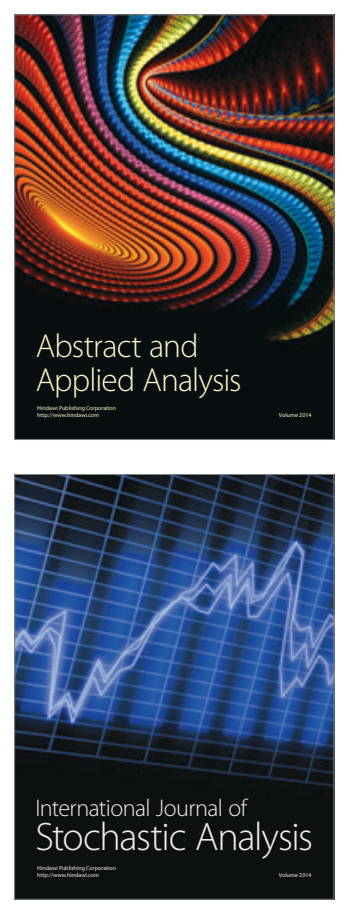

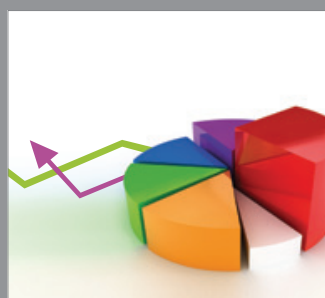

ournal of

Probability and Statistics

Promensencen
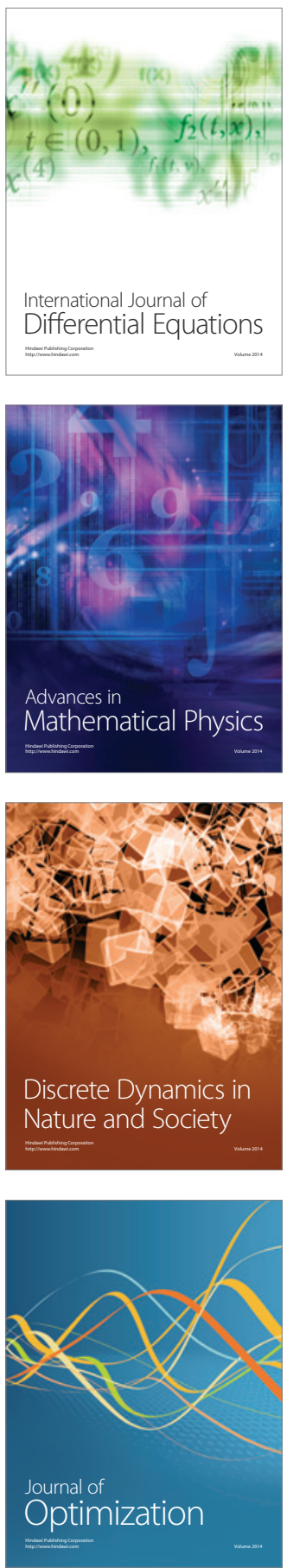